\title{
SPHARMA approximations for stationary functional time series on the sphere
}

\author{
Alessia Caponera ${ }^{1,2}$ \\ Received: 6 October 2020 / Accepted: 6 May 2021 / Published online: 12 May 2021 \\ (c) The Author(s) 2021
}

\begin{abstract}
In this paper, we focus on isotropic and stationary sphere-cross-time random fields. We first introduce the class of spherical functional autoregressive-moving average processes (SPHARMA), which extend in a natural way the spherical functional autoregressions (SPHAR) recently studied in Caponera and Marinucci (Ann Stat 49(1):346-369, 2021) and Caponera et al. (Stoch Process Appl 137:167-199, 2021); more importantly, we then show that SPHAR and SPHARMA processes of sufficiently large order can be exploited to approximate every isotropic and stationary sphere-cross-time random field, thus generalizing to this infinite-dimensional framework some classical results on real-valued stationary processes. Further characterizations in terms of functional spectral representation theorems and Woldlike decompositions are also established.
\end{abstract}

Keywords Time-varying spherical random fields · Functional time series · Double spectral representation · Spherical harmonics · Spherical functional ARMA

Mathematics Subject Classification 62M15 - 62M10 - 60G15 · 60F05 - 62M40 - 60G60

\section{Introduction}

Over the last few years, the analysis of sphere-cross-time isotropic and stationary random fields has drawn a considerable amount of attention, due to strong motivations arising in Climate and Atmospheric Sciences, Geophysics, Astrophysics and Cosmology, and many other areas of research, see for instance (Castruccio 2016; Castruccio and Stein 2013; Christakos 2005; Leonenko et al. 2018; Porcu et al. 2018, 2016; White and Porcu 2019) and the references therein. A lot of efforts has been spent, in particular, on the characterization of covariance functions and their use for parametric inference, see Berg and Porcu (2017), Gneiting (2002), Jeong and Jun (2015), Jun (2014), Porcu et al. (2018), Porcu et al. (2016) and White and Porcu (2019) and in particular Porcu et al. (2020) for a comprehensive review.

Alessia Caponera

alessia.caponera@epfl.ch

1 Dipartimento di Matematica, Università di Roma Tor Vergata, Rome, Italy

2 Institut de Mathématiques, Ecole Polytechnique Fédérale de Lausanne, Lausanne, Switzerland 
More recently, spherical functional autoregressive models (SPHAR) have been introduced and studied in Caponera and Marinucci (2021) and Caponera et al. (2021), where they were shown to provide a flexible tool for the analysis of time-dependent spherical data. In particular, in Caponera and Marinucci (2021) estimators based on a functional $L^{2}$-minimization criterion have been investigated, whereas in Caponera et al. (2021) LASSO-type penalized extensions were considered; indeed, various forms of concentration properties, laws of large numbers, quantitative and functional central limit theorems were established under broad general assumptions.

The purpose of the present work is to show that the spherical autoregression framework, and its natural generalization to spherical autoregressive moving averages (SPHARMA), provides a very general approximation for every isotropic and stationary sphere-cross-time random field. In particular, after introducing rigorously the SPHARMA class we show in our main results below (Theorems 1, 2 and 3) that any isotropic and stationary spherical random field can be approximated, in terms of their harmonic transforms and in the $L^{2}(\Omega)$ sense, by a SPHARMA or SPHAR process of sufficiently large order.

Of course, sphere-cross-time data can also be viewed as functional time series. In this sense, we can exploit the rich machinery recently developed in this area; the reader is referred to Bosq (2000), Bosq and Blanke (2007), Horváth and Kokoszka (2012), Hsing and Eubank (2015) and Ramsay and Silverman (2005) for an overview. In particular, as for the finite-dimensional case, the analysis of second-order properties is a crucial tool in the characterization of stationary functional processes. More precisely, some recent works have focused on a functional frequency-domain approach, encoding the complete second-order structure of stationary sequences, via the spectral density operator, i.e. the Fourier transform of the collection of autocovariance operators $\left\{\mathscr{R}_{t}, t \in \mathbb{Z}\right\}$,

$$
\mathscr{F}_{\lambda}:=\frac{1}{2 \pi} \sum_{t \in \mathbb{Z}} e^{-i \lambda t \mathscr{R}_{t}, \quad \lambda \in[-\pi, \pi] ;}
$$

all rigorous definitions will be given in Sect. 2. Such operators were first investigated in Panaretos and Tavakoli (2013b); then, Panaretos and Tavakoli (2013a) derived a CramérKarhunen-Loève representation for short-memory processes; we specialize this result to the spherical case to make this paper self-contained and complete. Loosely speaking, this representation first decomposes the series into an integral of uncorrelated frequency components (Cramér representation), each of which is in turn expanded in a Karhunen-Loève series, by means of eigenfunctions of $\mathscr{F}_{\lambda}$; this way at the same time it provides a full description of the second-order dynamics and it gives some insights into an optimal finite-dimensional representation. Very recently, van Delft and Eichler (2020) establish the functional versions of Herglotz's Theorem and the Cramér representation under more general assumptions, which cover also stationary Hilbert-valued time series with discontinuities in the spectral measure and long-memory processes. Overall, such results laid the foundations for a variety of frequency domain-based inference procedures in functional time series, e.g. Aue and van Delft (2020) and Hörmann et al. (2018).

Following these lines, we start with the investigation of the spectral characteristics of time-varying isotropic spherical random fields; under these circumstances it is possible to derive a double spectral decomposition based on spherical harmonics. Then, we focus on harmonic properties of functional autoregressive-moving average processes [see Bosq (2000) and Caponera and Marinucci (2021)], defined as random elements of $L^{2}\left(\mathbb{S}^{2}\right)$ (as mentioned above, recently Caponera and Marinucci (2021) and Caponera et al. (2021) have addressed the estimation of the functional linear autoregressive operators). Our approach is related to the 
extensive literature on linear stationary functional processes; however most of this literature is built on the estimation of autocovariance operators in the time domain, see among others (Bosq 2002; Dehling and Sharipov 2005; Mas 2002).

After building this background, we arrive at our main results; as anticipated, we prove that, under very broad assumptions, any isotropic and stationary spherical process can be approximated arbitrary well by a SPHARMA model of sufficiently large degree. Our results can be viewed as the infinite-dimensional counterpart of the rational approximations of spectral densities and the Wold decomposition in the analysis of standard, real-valued stationary processes, see for instance [Brockwell and Davis (1991), Chapters 4 and 5].

Plan of the paper. The paper is organized as follows. In Sect. 2, we describe the spectral properties of isotropic stationary sphere-cross-time random fields, while in Sect. 3 we provide a further characterization in terms functional spectral representation theorems. In Sect. 4, we introduce rigorously the SPHARMA class. Section 5 contains the main results of this work, that is, SPHARMA approximations of stationary and isotropic spherical random field, in terms of their harmonic transforms and in the $L^{2}(\Omega)$ sense, together with a Wold-like decomposition. Lastly, Sect. 6 collects the proofs.

Notation. We will use $\mathfrak{B}\left(\mathbb{S}^{2}\right)$ to denote the Borel $\sigma$-field on the unit sphere, $S O(3)$ to denote the special group of rotations in $\mathbb{R}^{3}$ [see Marinucci and Peccati (2011)], and $d$ to denote the Lebesgue measure. $L^{2}\left(\mathbb{S}^{2} ; \mathbb{C}\right):=L^{2}\left(\mathbb{S}^{2}, d x ; \mathbb{C}\right)$ will indicate the Hilbert space of square-integrable complex-valued functions on $\mathbb{S}^{2}$ endowed with the usual inner product $\langle f, g\rangle_{L^{2}\left(\mathbb{S}^{2} ; \mathbb{C}\right)}=\int_{\mathbb{S}^{2}} f(x) \overline{g(x)} d x$, whereas $\|\cdot\|_{L^{2}\left(\mathbb{S}^{2} ; \mathbb{C}\right)}$ will be the norm induced by $\langle\cdot, \cdot\rangle_{L^{2}\left(\mathbb{S}^{2} ; \mathbb{C}\right)}$; to simplify the notation, sometimes we will replace the subscript $L^{2}\left(\mathbb{S}^{2} ; \mathbb{C}\right)$ with 2 . Moreover, the restriction of $L^{2}\left(\mathbb{S}^{2} ; \mathbb{C}\right)$ to real-valued functions will be denoted by $L^{2}\left(\mathbb{S}^{2} ; \mathbb{R}\right)$. We will also use the same notation for $L^{2}\left(\mathbb{S}^{2} \times \mathbb{S}^{2} ; \mathbb{C}\right)$ and $L^{2}\left(\mathbb{S}^{2} \times \mathbb{S}^{2} ; \mathbb{R}\right)$. Let $\mathbb{H}$ be the Hilbert space of $L^{2}\left(\mathbb{S}^{2} ; \mathbb{C}\right)$-valued random elements with finite second moment, that is, $f \in \mathbb{H}$ is such that $\mathbb{E}\|f\|_{\mathbb{H}}^{2}<\infty$. The associated inner product is defined as $\langle f, g\rangle_{\mathbb{H}}=\mathbb{E}\langle f, g\rangle_{L^{2}\left(\mathbb{S}^{2} ; \mathbb{C}\right)}$, for $f, g \in \mathbb{H}$. Similarly, $L^{2}(\Omega):=L^{2}(\Omega, \mathbb{P} ; \mathbb{C})$ is the Hilbert space of complex-valued random variables with finite second moment, where $\mathbb{P}$ is the underlying probability measure. For $u, v \in L^{2}\left(\mathbb{S}^{2} ; \mathbb{C}\right)$, the tensor product $u \otimes v$ is defined to be the mapping that takes any element $f \in L^{2}\left(\mathbb{S}^{2} ; \mathbb{C}\right)$ to $u\langle f, v\rangle \in L^{2}\left(\mathbb{S}^{2} ; \mathbb{C}\right)$. $\|\mathscr{T}\|_{\mathrm{TR}}$ is the trace (or nuclear) norm of the operator $\mathscr{T}$, see Hsing and Eubank (2015). For a real- or complex-valued function $f$ defined on a set $D$, we define $\|f\|_{\infty}:=\sup _{x \in D}|f(x)|$; if not specified, $D=\mathbb{S}^{2} \times \mathbb{S}^{2} . \delta_{a}^{b}$ is the Kronecker delta function.

\section{Spectral characteristics}

Consider the collection of random variables $\left\{T(x, t),(x, t) \in \mathbb{S}^{2} \times \mathbb{Z}\right\}$ defined on the probability space $(\Omega, \mathfrak{F}, \mathbb{P})$. For every fixed $t \in \mathbb{Z},\left\{T(x, t), x \in \mathbb{S}^{2}\right\}$ is a spherical random field as defined in (Marinucci and Peccati 2011, Chapter 5); recall that we are implicitly assuming measurability with respect to the product $\sigma$-field $\mathfrak{B}\left(\mathbb{S}^{2}\right) \times \mathfrak{F}$. We name $\left\{T(x, t),(x, t) \in \mathbb{S}^{2} \times \mathbb{Z}\right\}$ space-time spherical random field. For simplicity, we will assume that $\mathbb{E}[T(x, t)]=0$, for all $(x, t) \in \mathbb{S}^{2} \times \mathbb{Z}$.

For the rest of the paper, we are going to consider space-time spherical random fields which are jointly isotropic (in the spatial component) and stationary (in the temporal component). To this purpose, we give the following definition [see also De la Cerda et al. (2018)]. 
Definition 1 We say that the zero-mean space-time spherical random field $\{T(x, t),(x, t) \in$ $\left.\mathbb{S}^{2} \times \mathbb{Z}\right\}$ is isotropic stationary if $\mathbb{E}|T(x, t)|^{2}<\infty$, for all $(x, t) \in \mathbb{S}^{2} \times \mathbb{Z}$, and

$$
\mathbb{E}[T(x, t) T(y, s)]=\mathbb{E}[T(g x, t+h) T(g y, s+h)],
$$

for all $x, y \in \mathbb{S}^{2}, g \in S O(3), t, s, h \in \mathbb{Z}$.

Thus, in this case we can define the autocovariance kernel at lag $t$

$$
r_{t}(x, y):=\mathbb{E}[T(x, t) T(y, 0)], \quad x, y \in \mathbb{S}^{2}, t \in \mathbb{Z},
$$

which is also Hilbert-Schmidt, i.e. $r_{t}(\cdot, \cdot) \in L^{2}\left(\mathbb{S}^{2} \times \mathbb{S}^{2} ; \mathbb{R}\right)$, and the corresponding operator $\mathscr{R}_{t}: L^{2}\left(\mathbb{S}^{2} ; \mathbb{C}\right) \rightarrow L^{2}\left(\mathbb{S}^{2} ; \mathbb{C}\right)$ induced by right integration, the autocovariance operator at lag $t$,

$$
\left(\mathscr{R}_{t} u\right)(\cdot)=\int_{\mathbb{S}^{2}} r_{t}(\cdot, y) u(y) d y, \quad u \in L^{2}\left(\mathbb{S}^{2} ; \mathbb{C}\right) .
$$

Note also that the autocovariance kernels are truly functions of $\langle x, y\rangle$, the standard inner product in $\mathbb{R}^{3}$. Indeed, $r_{t}(x, y)=r_{t}\left(x^{\prime}, y^{\prime}\right)$ for all pairs $(x, y),\left(x^{\prime}, y^{\prime}\right) \in \mathbb{S}^{2} \times \mathbb{S}^{2}$ such that $\langle x, y\rangle=\left\langle x^{\prime}, y^{\prime}\right\rangle$. Hence, we can argue that $E|T(x, t)|^{2}$ is constant in both space and time and the autocovariance kernels are uniformly bounded. Moreover, it can be shown that the induced autocovariance operators satisfy

$$
\mathscr{R}_{t} Y_{\ell, m}=C_{\ell}(t) Y_{\ell, m},
$$

that is, the $Y_{\ell, m}$ 's are eigenfunctions of $\mathscr{R}_{t}$ and the $C_{\ell}(t)$ 's are the associated eigenvalues [see Michel (2013), Theorem 7.3 and Corollary 7.4]. Consequently, the autocovariance kernels can be expanded in $L^{2}\left(\mathbb{S}^{2} \times \mathbb{S}^{2} ; \mathbb{C}\right)$ as

$$
r_{t}(x, y)=\sum_{\ell=0}^{\infty} C_{\ell}(t) \sum_{m=-\ell}^{\ell} Y_{\ell, m}(x) \overline{Y_{\ell, m}}(y)=\sum_{\ell=0}^{\infty} C_{\ell}(t) \frac{2 \ell+1}{4 \pi} P_{\ell}(\langle x, y\rangle),
$$

where the last equality comes from the so-called addition formula [see (Marinucci and Peccati 2011, p. 66)] and $P_{\ell}(\cdot)$ denotes the $\ell$-th Legendre polynomial (Szegö 1975, Sect. 4.7).

In functional data analysis, it is usual to model random processes as random elements of some separable Hilbert space. Under the joint isotropy-stationarity assumption, the sequences of spherical random fields we are considering can be seen as a sequence of random elements of $L^{2}\left(\mathbb{S}^{2} ; \mathbb{R}\right)$. More formally, for any fixed $t \in \mathbb{Z}$, there exists a random element $T_{t}$ of $L^{2}\left(\mathbb{S}^{2} ; \mathbb{R}\right)$ such that $T(\cdot, t)=T_{t} \mathbb{P}-$ a.s.; indeed

$$
\mathbb{E} \int_{\mathbb{S}^{2}}|T(x, t)|^{2} d x=4 \pi \mathbb{E}\left|T\left(x_{0}, t\right)\right|^{2} d x<\infty,
$$

where $x_{0}$ is any given point lying on $\mathbb{S}^{2}$. This implies that there exists a $\mathfrak{F}$-measurable set $\Omega^{\prime}$ of $\mathbb{P}$-probability 1 such that, for every $\omega \in \Omega^{\prime}, T(\cdot, t, \omega)$ is an element of $L^{2}\left(\mathbb{S}^{2} ; \mathbb{R}\right)$.

Remark 1 Recall that if $\left\{T(x), x \in \mathbb{S}^{2}\right\}$ is jointly measurable and $T(\cdot, \omega) \in L^{2}\left(\mathbb{S}^{2} ; \mathbb{R}\right)$ for each $\omega$, then the mapping $\omega \mapsto T(\cdot, \omega)$ is a random element of $L^{2}\left(\mathbb{S}^{2} ; \mathbb{R}\right.$ ) [see (Hsing and Eubank 2015, Theorem 7.4.1)].

Thus, $\left\{T_{t}, t \in \mathbb{Z}\right\}$ is a stationary sequence of random elements in $L^{2}\left(\mathbb{S}^{2} ; \mathbb{R}\right)$, with mean zero and $\mathbb{E}\left\|T_{0}\right\|_{2}^{2}<\infty$; see for instance (Bosq 2000, Definition 2.4) and $\mathscr{R}_{t}$ coincides with the autocovariance operator defined as the Bochner integral

$$
\mathbb{E}\left[T_{t} \otimes T_{0}\right]:=\int_{\Omega} T_{t} \otimes T_{0} d \mathbb{P} .
$$


In addition, $\mathbb{E}\left\|T_{0}\right\|_{2}^{2}=\left\|\mathscr{R}_{0}\right\|_{\mathrm{TR}}$.

In this setup, it is possible to show that the following representation holds

$$
T(x, t)=\sum_{\ell=0}^{\infty} \sum_{m=-\ell}^{\ell} a_{\ell, m}(t) Y_{\ell, m}(x),
$$

in the $L^{2}(\Omega)$ sense for every $(x, t) \in \mathbb{S}^{2} \times \mathbb{Z}$ and in the $L^{2}\left(\mathbb{S}^{2} \times \Omega\right)$ sense for every $t \in \mathbb{Z}$; the sequence $\left\{Y_{\ell, m}, \ell \geq 0, m=-\ell, \ldots, \ell\right\}$ is a standard orthonormal basis for $L^{2}\left(\mathbb{S}^{2} ; \mathbb{C}\right)$ of spherical harmonics, whereas, for fixed $t \in \mathbb{Z},\left\{a_{\ell, m}(t), \ell \geq 0, m=-\ell, \ldots, \ell\right\}$ is a triangular array of zero-mean uncorrelated random coefficients defined as

$$
a_{\ell, m}(t)=\int_{\mathbb{S}^{2}} T(x, t) \overline{Y_{\ell, m}(x)} d x .
$$

This result can be understood as a direct application of the spectral theorem for isotropic random fields on the sphere, see (Marinucci and Peccati 2011, Chapter 5 and in particular Theorem 5.13). In this sense, it does not give insights on the temporal dynamics of the process and, hence, on its complete second-order structure. However, it represents the starting point for further developments in this direction, as for instance the following propositions.

Proposition 1 For every fixed $(\ell, m),\left\{a_{\ell, m}(t), t \in \mathbb{Z}\right\}$ forms a zero-mean complex-valued stationary sequence and

$$
\mathbb{E}\left[a_{\ell, m}(t) \overline{a_{\ell^{\prime}, m^{\prime}}}(s)\right]=C_{\ell}(t-s) \delta_{\ell}^{\ell^{\prime}} \delta_{m}^{m^{\prime}}, \quad t, s \in \mathbb{Z} .
$$

Proposition 2 The covariance kernels (1) of a zero-mean isotropic stationary space-time spherical random field $\left\{T(x, t),(x, t) \in \mathbb{S}^{2} \times \mathbb{Z}\right\}$ are continuous on $\mathbb{S}^{2} \times \mathbb{S}^{2}$ and the convergence in (3) is uniform.

Remark 2 A careful inspection of the proof of Proposition 2 reveals that, for a spherical random field $\left\{T(x), x \in \mathbb{S}^{2}\right\}$, mean-square continuity can be proved by relaxing the hypothesis in Marinucci and Peccati (2013), from strong isotropy to (2-weak) isotropy; here we refer to (Marinucci and Peccati 2011, Definition 5.9).

Remark 3 Following the works Schoenberg (1942) and Gneiting (2013), in Berg and Porcu (2017) the authors give a mathematical characterization of covariance functions for isotropic stationary random fields over $\mathbb{S}^{2} \times \mathbb{R}$. In De la Cerda et al. (2018) the regularity properties of such covariance functions have been investigated for the case where a double KarhunenLoève expansion holds. Examples of random fields satisfying this decomposition are found in the Appendix of Porcu et al. (2016).

In line with Panaretos and Tavakoli (2013b), we shall use the conditions below to define the spectral density kernels and the spectral density operators and to prove part of our main results in Sect. 5. Under these conditions, we are also able to give first a Functional Cramér Representation which involves a $L^{2}\left(\mathbb{S}^{2} ; \mathbb{C}\right.$ )-valued orthogonal increment process, and then to obtain a double spectral representation with respect to both space and time, see Sect. 3 below. We stress that in this section and in Sect. 3, as in Panaretos and Tavakoli (2013a), it is not assumed any other prior structural properties for the stationary sequence (e.g., linearity or Gaussianity).

Condition 1 For an isotropic stationary space-time spherical random field $\{T(x, t),(x, t) \in$ $\left.\mathbb{S}^{2} \times \mathbb{Z}\right\}$ with covariance kernels (1), consider one of the following conditions: 
(i) the autocovariance kernels satisfy $\sum_{t \in \mathbb{Z}}\left\|r_{t}\right\|_{2}<\infty$;

(ii) the autocovariance operators satisfy $\sum_{t \in \mathbb{Z}}\left\|\mathscr{R}_{t}\right\|_{\mathrm{TR}}<\infty$.

It is readily seen that Condition ( $i i)$ implies $(i)$.

In Panaretos and Tavakoli (2013b) there is an extensive discussion on the role of such assumptions. Similarly here, under Condition $1(i)$, it is possible to define the spectral density kernel at frequency $\lambda \in[-\pi, \pi]$,

$$
f_{\lambda}(x, y):=\frac{1}{2 \pi} \sum_{t \in \mathbb{Z}} e^{-i \lambda t} r_{t}(x, y),
$$

where the convergence is in $\|\cdot\|_{2}$. It is uniformly bounded and also uniformly continuous in $\lambda$ with respect to $\|\cdot\|_{2}$. The spectral density operator $\mathscr{F} \lambda: L^{2}\left(\mathbb{S}^{2} ; \mathbb{C}\right) \rightarrow L^{2}\left(\mathbb{S}^{2} ; \mathbb{C}\right)$, the operator induced by the spectral density kernel through right-integration, is self-adjoint and nonnegative definite for all $\lambda \in[-\pi, \pi]$. Moreover, the following inversion formula holds in the $L^{2}$ sense:

$$
\int_{0}^{2 \pi} f_{\alpha}(x, y) e^{i t \alpha} d \alpha=r_{t}(x, y) .
$$

The previous results can also be reformulated in terms of $\|\cdot\|_{\infty}$ if one has $\sum_{t \in \mathbb{Z}}\left\|r_{t}\right\|_{\infty}<$ $\infty$. In this case, continuity of autocovariance kernels implies continuity of spectral density kernels.

Under Condition 1 ( $\mathrm{i}$ i), we have an alternative representation for the spectral density operator at frequency $\lambda \in[-\pi, \pi]$, i.e.,

$$
\mathscr{F}_{\lambda}=\frac{1}{2 \pi} \sum_{t \in \mathbb{Z}} e^{-i \lambda t} \mathscr{R}_{t},
$$

where the convergence holds in nuclear norm. In particular, $\mathscr{F} \lambda$, seen as a function of $\lambda$, is uniformly bounded and uniformly continuous with respect to $\|\cdot\|_{\mathrm{TR}}$.

The reader is referred to Panaretos and Tavakoli (2013b) for proofs of these assertions.

Remark 4 Condition 1 is strictly related to the concept of short memory stationary processes; indeed, stationary processes which exhibit short-range dependence are those with absolutely summable autocovariances and, hence, bounded and continuous spectral density function, e.g., stationary ARMA processes. For functional time series, this translates into absolutely summable autocovariance operators and bounded and continuous spectral density operator, that is, their norms are, respectively, absolutely summable, and bounded and continuous.

Exploiting joint isotropy-stationarity of the space-time spherical random field, we can specialize all the previous results and obtain a neat expression for our quantities of interest. First of all, in this setting, Condition 1 ( $i i$ ) implies $\sum_{t \in \mathbb{Z}}\left\|r_{t}\right\|_{\infty}<\infty$, which implies $(i)$. In all three cases, one has

$$
\mathscr{F}_{\lambda} Y_{\ell, m}=\left(\frac{1}{2 \pi} \sum_{t \in \mathbb{Z}} e^{-i \lambda t} C_{\ell}(t)\right) Y_{\ell, m} .
$$

We can then define the eigenvalues $f_{\ell}(\lambda):=\frac{1}{2 \pi} \sum_{t \in \mathbb{Z}} e^{-i t \lambda} C_{\ell}(t)$ and expand the spectral density kernels in terms of Legendre polynomials, i.e.,

$$
f_{\lambda}(x, y)=\sum_{\ell=0}^{\infty} f_{\ell}(\lambda) \frac{2 \ell+1}{4 \pi} P_{\ell}(\langle x, y\rangle),
$$


where the convergence holds in $\|\cdot\|_{2}$ under Condition 1 ( $i$ ), and in $\|\cdot\|_{\infty}$ under $(i i)$ since $f_{\lambda}(\cdot, \cdot)$ is continuous on $\mathbb{S}^{2} \times \mathbb{S}^{2}$ and

$$
\left\|\mathscr{F}_{\lambda}\right\|_{\mathrm{TR}}=\sum_{\ell=0}^{\infty}(2 \ell+1) f_{\ell}(\lambda)<\infty .
$$

In addition, the eigenvalues $f_{\ell}(\lambda)$ are uniformly bounded and uniformly continuous in $\lambda$. Indeed,

$$
0 \leq f_{\ell}(\lambda) \leq\left\|f_{\lambda}\right\|_{2} \leq \sum_{t \in \mathbb{Z}}\left\|r_{t}\right\|_{2}, \quad \text { for all } \lambda,
$$

and, given $\epsilon>0$, there exists $\delta>0$ such that

$$
\left|\lambda_{1}-\lambda_{2}\right|>\delta \Longrightarrow\left|f_{\ell}\left(\lambda_{1}\right)-f_{\ell}\left(\lambda_{2}\right)\right| \leq\left\|f_{\lambda_{1}}-f_{\lambda_{2}}\right\|_{2}<\epsilon .
$$

\section{Spectral representations}

This section builds on the earlier works (Panaretos and Tavakoli 2013a,b) and it provides some results on a double spectral representation, with respect to both the temporal and spatial components of the field. The main purpose is to study these objects, trying to simultaneously capture the surface structure (spatial component) as well as the dynamics in time (temporal component); what in Panaretos and Tavakoli (2013a) is called within/between curve dynamics. We then specilize the results in Panaretos and Tavakoli (2013a), for dependent random functions on the interval $[0,1]$, to the case of the sphere, making this paper self-contained and complete.

The following proposition is the analogue of Theorem 2.1 in Panaretos and Tavakoli (2013a) and it can be seen as the infinite-dimensional version of the well-known spectral representation of real-valued stationary processes.

Proposition 3 (Spherical Functional Cramér Representation) Under Condition 1 (ii), $T_{t}$ admits the representation

$$
T_{t}=\int_{-\pi}^{\pi} e^{i t \lambda} d Z_{\lambda}, \quad \text { a.s. in } L^{2}\left(\mathbb{S}^{2} ; \mathbb{C}\right)
$$

where, for fixed $\lambda, Z_{\lambda}$ is a random element of $L^{2}\left(\mathbb{S}^{2} ; \mathbb{C}\right)$ with $\mathbb{E}\left\|Z_{\lambda}\right\|_{2}^{2}=\sum_{\ell=0}^{\infty}(2 \ell+$ 1) $\int_{-\pi}^{\lambda} f_{\ell}(\nu) d v$, and the process $\left\{Z_{\lambda},-\pi \leq \lambda \leq \pi\right\}$ has orthogonal increments:

$$
\mathbb{E}\left\langle Z_{\lambda_{1}}-Z_{\lambda_{2}}, Z_{\lambda_{3}}-Z_{\lambda_{4}}\right)_{2}=0, \quad \lambda_{1}>\lambda_{2} \geq \lambda_{3}>\lambda_{4}
$$

The representation (5) is called the Cramér representation of $T_{t}$, and the stochastic integral involved can be understood as a Riemann-Stieltjes limit, in the sense that

$$
\mathbb{E}\left\|T_{t}-\sum_{j=1}^{J} e^{i \lambda_{j} t}\left(Z_{\lambda_{j+1}}-Z_{\lambda_{j}}\right)\right\|_{L^{2}\left(\mathbb{S}^{2} ; \mathbb{C}\right)}^{2} \rightarrow 0, \quad J \rightarrow \infty,
$$

where $-\pi=\lambda_{1}<\cdots<\lambda_{J+1}=\pi$ and $\max _{j=1, \ldots, J}\left|\lambda_{j+1}-\lambda_{j}\right| \rightarrow 0$ as $J \rightarrow \infty$.

Now, we are going to establish a double spectral representation result, by showing the relation between the orthogonal increment process $\left\{\alpha_{\ell, m}(\lambda),-\pi \leq \lambda \leq \pi\right\}$ and $\left\{Z_{\lambda},-\pi \leq\right.$ 
$\lambda \leq \pi\}$. It is worth to notice that, under Condition 1, all the results presented in Panaretos and Tavakoli (2013a) can be easily extended to our framework, including the so-called CramérKarhunen-Loève Representation. Such a representation decomposes the space-time spherical random field into uncorrelated functional frequency components, exploiting an orthonormal basis for $L^{2}\left(\mathbb{S}^{2} ; \mathbb{C}\right)$ made up of eigenfunctions of the spectral density operator $\mathscr{F} \lambda$. However, in the anisotropic case, these eigenfunctions are unknown and have to be estimated. The stronger conditions allows to apply directly theorems from Panaretos and Tavakoli (2013a), since we have an explicit eigenvalue-eigenfunction decomposition of the spectral density operator in terms of spherical harmonics.

Proposition 4 (Spherical Cramér-Karhunen-Loève Representation) Under Condition 1 (ii), for every $t \in \mathbb{Z}$ and every $x \in \mathbb{S}^{2}$,

$$
\mathbb{E}\left|T(x, t)-\sum_{\ell=0}^{L} \sum_{m=-\ell}^{\ell} \int_{-\pi}^{\pi} e^{i t \lambda} d \alpha_{\ell, m}(\lambda) Y_{\ell, m}(x)\right|^{2} \rightarrow 0, \quad L \rightarrow \infty,
$$

with $\alpha_{\ell, m}(\lambda):=\left\langle Z_{\lambda}, Y_{\ell, m}\right\rangle_{2}$ and $Z_{\lambda}$ as defined in Proposition 3,

$$
\mathbb{E}\left[\alpha_{\ell, m}(\omega) \alpha_{\ell^{\prime}, m^{\prime}}(\beta)\right]=\int_{-\pi}^{\min (\omega, \beta)} f_{\ell}(\alpha) d \alpha \delta_{\ell}^{\ell^{\prime}} \delta_{m}^{m^{\prime}} .
$$

Remark 5 Note that the effective dimensionality of each frequency component is captured by the eigenvalues of the spectral density operators. The approximation error is then given by

$$
\mathbb{E}\left|T(x, t)-\sum_{\ell=0}^{L} \sum_{m=-\ell}^{\ell} \int_{-\pi}^{\pi} e^{i t \lambda} d \alpha_{\ell, m}(\lambda) Y_{\ell, m}(x)\right|^{2}=\sum_{\ell>L} \frac{2 \ell+1}{4 \pi} \int_{-\pi}^{\pi} f_{\ell}(\lambda) d \lambda,
$$

see also (Panaretos and Tavakoli 2013a, Remark 3.10).

\section{Spherical functional ARMA}

In this section, we extend the spherical functional autoregressions (SPHAR), first introduced in Caponera and Marinucci (2021), to include a moving-average term in the error. This leads to the definition of the so-called spherical functional autoregressive-moving average processes (SPHARMA). The main purpose here is to study the existence and uniqueness of an isotropic stationary solution of the functional autoregressive-moving average equation, see also (Bosq 2000, Chapter 5).

We first recall the definitions of spherical white noise and isotropic kernel operator, see Caponera and Marinucci (2021) and Caponera et al. (2021).

Definition 2 The collection of random variables $\left\{Z(x, t),(x, t) \in \mathbb{S}^{2} \times \mathbb{Z}\right\}$ is said to be a spherical white noise if:

- for every fixed $t \in \mathbb{Z},\left\{Z(x, t), x \in \mathbb{S}^{2}\right\}$ is a zero-mean isotropic random field, with covariance kernel

$$
r_{Z}(x, y)=\sum_{\ell=0}^{\infty} \frac{2 \ell+1}{4 \pi} C_{\ell ; Z} P_{\ell}(\langle x, y\rangle), \quad \sum_{\ell=0}^{\infty} \frac{2 \ell+1}{4 \pi} C_{\ell ; Z}<\infty,
$$

$\left\{C_{\ell ; Z}\right\}$ denoting as usual the angular power spectrum of $Z(\cdot, t)$; 
- for every $t \neq s, \mathbb{E}\left[a_{\ell, m ; Z}(t) \overline{a_{\ell^{\prime}, m^{\prime} ; Z}}(s)\right]=0$, for all $\ell, \ell^{\prime} \geq 0,|m| \leq \ell,\left|m^{\prime}\right| \leq \ell^{\prime}$, where

$$
a_{\ell, m ; Z}(t)=\int_{\mathbb{S}^{2}} Z(x, t) \overline{Y_{\ell, m}}(x) d x .
$$

We shall write $Z \sim S W N\left(0,\left\{C_{\ell ; Z}\right\}\right)$. Moreover, $\left\{Z(x, t),(x, t) \in \mathbb{S}^{2} \times \mathbb{Z}\right\}$ is said to be a strong spherical white noise if it satisfies $(i)$ and the random fields $\left\{Z(x, t), x \in \mathbb{S}^{2}\right\}, t \in \mathbb{Z}$, are independent and identically distributed.

Definition 3 A spherical isotropic kernel operator is an application $\Phi: L^{2}\left(\mathbb{S}^{2}\right) \rightarrow L^{2}\left(\mathbb{S}^{2}\right)$ which satisfies

$$
(\Phi f)(x)=\int_{\mathbb{S}^{2}} k(\langle x, y\rangle) f(y) d y, \quad x \in \mathbb{S}^{2},
$$

for some continuous $k:[-1,1] \rightarrow \mathbb{R}$.

The following representation holds in the $L^{2}$-sense for the kernel associated with $\Phi$ :

$$
k(\langle x, y\rangle)=\sum_{\ell=0}^{\infty} \phi_{\ell} \frac{2 \ell+1}{4 \pi} P_{\ell}(\langle x, y\rangle) .
$$

The coefficients $\left\{\phi_{\ell}, \ell \geq 0\right\}$ corresponds to the eigenvalues of the operator $\Phi$ and the associated eigenfunctions are the family of spherical harmonics $\left\{Y_{\ell, m}\right\}$, yielding

$$
\Phi Y_{\ell, m}=\phi_{\ell} Y_{\ell, m} .
$$

Thus, it holds $\sum_{\ell}(2 \ell+1)\left|\phi_{\ell}\right|^{2}<\infty$, and hence this operator is Hilbert-Schmidt [see, e.g., Hsing and Eubank (2015)]. In Caponera and Marinucci (2021) and Caponera et al. (2021), the authors also consider trace class operators, namely, such that $\sum_{\ell}(2 \ell+1)\left|\phi_{\ell}\right|<\infty$, for which the representation (7) holds uniformly.

Now, we focus on a space-time spherical random field $\left\{T(x, t),(x, t) \in \mathbb{S}^{2} \times \mathbb{Z}\right\}$, as defined in Sect. 1 , for which it holds almost surely $T(\cdot, t) \in L^{2}\left(\mathbb{S}^{2} ; \mathbb{R}\right), t \in \mathbb{Z}$.

Definition $4\left\{T(x, t),(x, t) \in \mathbb{S}^{2} \times \mathbb{Z}\right\}$ is said to be a $\operatorname{SPHARMA}(p, q)$ process if there exist $p$ isotropic kernel operators $\left\{\Phi_{1}, \ldots, \Phi_{p}\right\}, q$ isotropic kernel operators $\left\{\Theta_{1}, \ldots, \Theta_{q}\right\}$ and a spherical white noise $\left\{Z(x, t),(x, t) \in \mathbb{S}^{2} \times \mathbb{Z}\right\}$ such that

$$
T(x, t)-\sum_{j=1}^{p}\left(\Phi_{j} T(\cdot, t-j)\right)(x)=Z(x, t)+\sum_{j=1}^{q}\left(\Theta_{j} Z(\cdot, t-j)\right)(x),
$$

for all $(x, t) \in \mathbb{S}^{2} \times \mathbb{Z}$, the equality holding both in the $L^{2}(\Omega)$ and in the $L^{2}\left(\mathbb{S}^{2} \times \Omega\right)$ sense.

Remark 6 Note that, following Caponera and Marinucci (2021), the solution process (as well as the spherical white noise) is defined pointwise, i.e., for each $(x, t)$ there exists a random

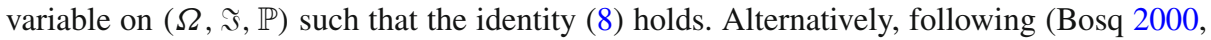
p. 72), one could give the definition in terms of random elements of $L^{2}\left(\mathbb{S}^{2} ; \mathbb{R}\right)$. The two approaches are actually equivalent, because we are dealing with jointly-measurable meansquare continuous random fields.

Similarly to Caponera and Marinucci (2021) and Caponera et al. (2021), it is possible to write

$$
a_{\ell, m}(t)-\sum_{j=1}^{p} \phi_{\ell ; j} a_{\ell, m}(t-j)=a_{\ell, m ; Z}(t)+\sum_{j=1}^{q} \theta_{\ell ; j} a_{\ell, m ; Z}(t-j) ;
$$


where the coefficients $\left\{\phi_{\ell ; j}, \ell \geq 0, j=1, \ldots, p\right\}$ and $\left\{\theta_{\ell ; j}, \ell \geq 0, j=1, \ldots, q\right\}$ are respectively the eigenvalues of the operators $\left\{\Phi_{j}, j=1, \ldots, p\right\}$ and $\left\{\Theta_{j}, j=1, \ldots, q\right\}$.

Now, define the polynomials $\phi_{\ell}: \mathbb{C} \rightarrow \mathbb{C}$ and $\theta_{\ell}: \mathbb{C} \rightarrow \mathbb{C}, \ell \geq 0$, such that

$$
\phi_{\ell}(z)=1-\phi_{\ell ; 1} z-\cdots-\phi_{\ell ; p} z^{p}, \quad \theta_{\ell}(z)=1+\theta_{\ell ; 1} z-\cdots-\theta_{\ell ; q} z^{q} ;
$$

note that the actual degrees can change with $\ell$. Clearly, particular cases of the $\operatorname{SPHARMA}(p, q)$ process can be obtained by letting one of the two sequences constant and equal to 1 . For instance, if $\phi_{\ell}(z) \equiv 1$, for all $\ell \geq 0$, we obtain a spherical functional moving-average process of order $q$ (or SPHMA $(q)$ ), whereas, If $\theta_{\ell}(z) \equiv 1$, for all $\ell \geq 0$, then we have the socalled spherical functional autoregressive process of order $p$ (or $\operatorname{SPHAR}(p)$ ), see Caponera and Marinucci (2021) and Caponera et al. (2021).

Condition 2 (Causality/Stationarity) The two sequences of polynomials in Eq. (10) are such that $\phi_{\ell}(\cdot)$ and $\theta_{\ell}(\cdot)$ have no common zeroes and

$$
|z| \leq 1 \Rightarrow \phi_{\ell}(z) \neq 0 .
$$

More explicitly, there are no roots in the unit disk, for all $\ell \geq 0$.

Remark 7 We say that a SPHARMA $(p, q)$ process satisfying Condition 2 is causal. Similarly, if $|z| \leq 1 \Rightarrow \theta_{\ell}(z) \neq 0$, for all $\ell \geq 0$, we call it invertible.

Remark 8 Since $\sum_{\ell}(2 \ell+1)\left|\phi_{\ell ; j}\right|^{2}<\infty$ for all $j=1, \ldots, p$, Condition 2 actually ensures that there exists $\delta>0$ such that

$$
|z|<1+\delta \Rightarrow \phi_{\ell}(z) \neq 0, \quad \text { for all } \ell \geq 0 .
$$

Indeed, it is possible to show that if we consider all the polynomials $\phi_{\ell}(\cdot)$ of degree $d_{\ell} \neq 0$ with distinct roots $\xi_{\ell ; 1}, \ldots, \xi_{\ell ; r_{\ell}}$, then

$$
\left|\xi_{\ell ; j}\right| \geq \xi_{*}>1, \quad \text { uniformly over } \ell .
$$

Condition 3 (Identifiability) The spherical white noise process $\left\{Z(x, t),(x, t) \in \mathbb{S}^{2} \times \mathbb{Z}\right\}$ is such that $C_{\ell ; Z}>0$, for all $\ell \geq 0$.

Example 1 [SPHMA $(q)]\left\{T(x, t),(x, t) \in \mathbb{S}^{2} \times \mathbb{Z}\right\}$ is a spherical moving-average process of order $\mathrm{q}$ if for all pairs $(x, t) \in \mathbb{S}^{2} \times \mathbb{Z}$ it satisfies

$$
T(x, t)=Z(x, t)+\sum_{j=1}^{q}\left(\Theta_{j} Z(\cdot, t-j)\right)(x) .
$$

It is readily seen in this case that the difference equations have the unique solution (12). Moreover, the solution process is isotropic stationary since for $\ell \geq 0, m=-\ell, \ldots, \ell$, defining $\theta_{\ell ; 0}=1$ and $\theta_{\ell ; j}=0$ for $j>q$,

$$
\mathbb{E}\left[a_{\ell, m}(t)\right]=\sum_{j=0}^{q} \theta_{\ell ; j} \mathbb{E}\left[a_{\ell, m ; Z}(t)\right]=0,
$$

and

$$
\mathbb{E}\left[a_{\ell, m}(t+h) \overline{a_{\ell, m}}(t)\right]= \begin{cases}C_{\ell ; Z} \sum_{j=0}^{q-|h|} \theta_{\ell ; j} \theta_{\ell ; j+|h|} & \text { if }|h| \leq q, \\ 0 & \text { if }|h|>q\end{cases}
$$


Example 2 (SPHAR $(1))\left\{T(x, t),(x, t) \in \mathbb{S}^{2} \times \mathbb{Z}\right\}$ is a spherical autoregressive process of order one if for all pairs $(x, t) \in \mathbb{S}^{2} \times \mathbb{Z}$ it satisfies

$$
T(x, t)=\left(\Phi_{1} T(\cdot, t-1)\right)(x)+Z(x, t)
$$

in this case, Condition 2 simply becomes $\left|\phi_{\ell ; 1}\right|<1$, for all $\ell \geq 0$.

Differently from the SPHMA case, the existence and uniqueness of an isotropic stationary solution of the SPHAR (or SPHARMA) equation needs closer investigation. This is the aim of the following statement. The proof is given already in Bosq (2000) for the simplest case of order one Hilbert-valued autoregressive processes, but here we construct explicitly the solution with a slightly different argument for completeness.

Proposition 5 Under Conditions 2 and 3, the unique isotropic stationary solution to (8) is given by

$$
T(x, t)=\lim _{k \rightarrow \infty} T_{k}(x, t), \quad T_{k}(x, t)=\sum_{\ell=0}^{L_{k}} \sum_{m=-\ell}^{\ell} \sum_{j=0}^{k} \psi_{\ell ; j} a_{\ell m ; Z}(t-j) Y_{\ell m}(x),
$$

in the $L^{2}(\Omega)$ and $L^{2}\left(\mathbb{S}^{2} \times \Omega\right)$ sense. The coefficients $\left\{\psi_{\ell ; j}\right\}$ are determined by the relation

$$
\psi_{\ell}(z)=\sum_{j=0}^{\infty} \psi_{\ell ; j} z^{j}=\theta_{\ell}(z) / \phi_{\ell}(z), \quad|z| \leq 1 .
$$

The spectral density kernel at frequency $\lambda \in[-\pi, \pi]$ is well defined as the limit in $\|\cdot\|_{\infty}$

$$
f_{\lambda}(x, y):=\sum_{\ell=0}^{\infty} f_{\ell}(\lambda) \frac{2 \ell+1}{4 \pi} P_{\ell}(\langle x, y\rangle),
$$

with

$$
f_{\ell}(\lambda)=\frac{C_{\ell ; Z}}{2 \pi}\left|\psi_{\ell}\left(e^{i \lambda}\right)\right|^{2}, \quad \ell \geq 0 .
$$

$\mathscr{F}_{\lambda}$, the corresponding operator induced through right-integration, is uniformly bounded and uniformly continuous in $\lambda$ with respect to $\|\cdot\|_{\mathrm{TR}}$.

Remark 9 Notice that the isotropic stationary solutions of the SPHAR(1) Eq. (13) take the form

$$
T(\cdot, t)=\sum_{j=0}^{\infty} \Phi_{1}^{j} Z(\cdot, t)
$$

and Condition 2 is satisfied if and only if the operator norm $\left\|\Phi_{1}\right\|_{\text {op }}:=\max _{\ell \geq 0}\left|\phi_{\ell ; 1}\right|<1$, see also (Bosq 2000, Sect. 3.4).

Remark 10 Both Caponera and Marinucci (2021) and Caponera et al. (2021) introduce two estimation procedures for the spherical autoregressive kernels $\left\{k_{j}, j=1, \ldots, p\right\}$ and investigate asymptotic properties of the corresponding nonparametric estimators. Specifically, in Caponera and Marinucci (2021), the authors focus on the solutions of a functional $L^{2}$ minimization problem, while, in Caponera et al. (2021), they add a convex penalty term to study LASSO-type estimators under sparsity assumptions. The core idea behind these approaches is to transform a nonparametric kernel estimation problem into the investigation 
of a growing sequence of spectral parameters (precisely, the $\phi_{\ell ; j}$ 's). With this in mind, one can think of building a functional procedure for the full SPHARMA model by looking at the subprocesses (9). We refer the reader to (Brockwell and Davis 1991, Chapter 8) for an overview of standard techniques applied to finite-dimensional ARMA.

\section{Main results}

\subsection{SPHAR and SPHMA approximations of spectral density operators}

In what follows, we show that for any real-valued isotropic stationary random field, with spectral density kernels $f_{\lambda}$ satisfying Condition $1(i)$, it is possible to find both a causal $\operatorname{SPHAR}(p)$ process and an invertible $\operatorname{SPHMA}(q)$ process whose spectral density kernels are arbitrarily close to $f_{\lambda}$ in the $L^{2}$ norm. This suggests that the original process can be approximated in some sense by either a $\operatorname{SPHAR}(p)$ or a $\operatorname{SPHMA}(q)$ process. Similar results hold for the spectral density operator $\mathscr{F}_{\lambda}$ in the trace class norm under the stronger Condition 1 (ii).

Below we will denote with $T_{L}$ a band-limited space-time spherical random field, namely such that it can be expanded in terms of finitely many spherical harmonics, up to a finite multipole $\ell=L$.

Theorem 1 If $f_{\lambda}(\cdot, \cdot)$ is a spectral density kernel of an isotropic stationary process, satisfying Condition $1(i)$, then $\forall \epsilon>0$ there exists an invertible $\operatorname{SPHMA}(q)$ process

$$
\begin{aligned}
T_{L}(x, t)= & Z_{L}(x, t)+\left(\Theta_{1} Z_{L}(\cdot, t-1)\right)(x)+\ldots \\
& +\left(\Theta_{q} Z_{L}(\cdot, t-q)\right)(x), \quad Z_{L} \sim \operatorname{SWN}\left(0,\left\{\sigma_{\ell}^{2}\right\}\right),
\end{aligned}
$$

with spectral density kernel $\tilde{f}_{\lambda}(\cdot, \cdot)$ such that

$$
\left\|\tilde{f}_{\lambda}-f_{\lambda}\right\|_{2} \leq \epsilon \text { for all } \lambda \in[-\pi, \pi]
$$

where $\sigma_{\ell}^{2}=\left(1+\theta_{\ell ; 1}^{2}+\cdots+\theta_{\ell ; q}^{2}\right)^{-1} \int_{-\pi}^{\pi} f_{\ell}(\lambda) d \lambda, \ell=0, \ldots$, L. Under only Condition 1 (ii),

$$
\left\|\tilde{\mathscr{F}}_{\lambda}-\mathscr{F}_{\lambda}\right\|_{\mathrm{TR}} \leq \epsilon \text { for all } \lambda \in[-\pi, \pi]
$$

Theorem 2 If $f_{\lambda}(\cdot, \cdot)$ is a spectral density kernel of an isotropic stationary process, satisfying Condition $1(i)$, then $\forall \epsilon>0$ there exists a causal $\operatorname{SPHAR}(p)$ process

$$
\begin{aligned}
T_{L}(x, t)= & \left(\Phi_{1} T_{L}(\cdot, t-1)\right)(x)+\cdots+\left(\Phi_{p} T_{L}(\cdot, t-p)\right)(x) \\
& +Z_{L}(x, t), \quad Z_{L} \sim \operatorname{SWN}\left(0,\left\{\sigma_{\ell}^{2}\right\}\right),
\end{aligned}
$$

with spectral density kernel $\tilde{f}_{\lambda}(\cdot, \cdot)$ such that

$$
\left\|\tilde{f}_{\lambda}-f_{\lambda}\right\|_{2} \leq \epsilon \text { for all } \lambda \in[-\pi, \pi]
$$

Under only Condition 1 ( $i$ i ),

$$
\left\|\tilde{\mathscr{F}}_{\lambda}-\mathscr{F}_{\lambda}\right\|_{\mathrm{TR}} \leq \epsilon \text { for all } \lambda \in[-\pi, \pi] .
$$

Remark 11 Even from an inferential point of view, these results suggest a way to estimate the spectral density operator $\mathscr{F}_{\lambda}$ of any isotropic stationary sphere-cross-time random field. 
Indeed, assuming to be able to observe the projections of the fields on the spherical harmonics basis, it is possible to derive a sequence of rational estimators of the form

$$
\hat{f}_{\ell}(\lambda)=\frac{\hat{\sigma}_{\ell}}{2 \pi} \frac{\left|1+\hat{\theta}_{\ell ; 1} e^{-i \lambda}-\cdots-\hat{\theta}_{\ell ; q} e^{-i \lambda q}\right|^{2}}{\left|1-\hat{\phi}_{\ell ; 1} e^{-i \lambda}-\cdots-\hat{\phi}_{\ell ; p} e^{-i \lambda p}\right|^{2}}, \quad \ell \geq 0,
$$

for the eigenvalues characterizing $\mathscr{F}_{\lambda}$; see also (Brockwell and Davis 1991, Sect. 10.6).

\subsection{The $L^{2}(\Omega)$ approximations}

Here we prove that any stationary spherical functional process can be approximated in the $L^{2}(\Omega)$ sense by both a SPHMA process and a SPHAR process of sufficiently large degree. We also establish a functional Wold decomposition, which allows to represent the field as a sum of a linear process and a deterministic process, similarly to the finite-dimensional case, see (Brockwell and Davis 1991, Theorem 5.7.1) and also Bosq (2000); Bosq and Blanke (2007). This result is given in the auxiliary Lemma 1 and it is instrumental for the proof of our last main theorem.

More formally, consider as usual a zero-mean isotropic stationary process $\{T(x, t),(x, t) \in$ $\mathbb{S}^{2} \times \mathbb{Z}$ \}. For each $(\ell, m)$, define the sequence of closed linear subspaces of $L^{2}(\Omega)$

$$
\begin{aligned}
& \mathcal{M}_{\ell, m ; n}=\overline{\operatorname{span}\left\{a_{\ell, m}(n),-\infty<t \leq n\right\}}, \quad n \in \mathbb{Z}, \\
& \mathcal{M}_{\ell, m ;-\infty}=\bigcap_{n=-\infty}^{\infty} \mathcal{M}_{\ell, m ; n},
\end{aligned}
$$

and the $\ell$-th one-step mean squared error

$$
\sigma_{\ell}^{2}=\mathbb{E}\left|a_{\ell, m}(n)-P_{\mathcal{M}_{\ell, m ; n}} a_{\ell, m}(n+1)\right|^{2}=\inf _{f \in \mathcal{M}_{\ell, m ; n}} \mathbb{E}\left|a_{\ell, m}(n+1)-f\right|^{2},
$$

where $P_{\mathcal{M}_{\ell, m ; n}}$ is the projection operator on $\mathcal{M}_{\ell, m ; n}$, see (Brockwell and Davis 1991, Chapter 5). Note that

$$
\sigma^{2}=\sum_{\ell=0}^{\infty}(2 \ell+1) \sigma_{\ell}^{2}<\infty
$$

indeed, if we define $\mathcal{M}_{n}=\overline{\operatorname{span}\left\{T_{t},-\infty<t \leq n\right\}} \subset \mathbb{H}$, we can observe that

$$
\infty>\mathbb{E}\left\|T_{n+1}-P_{\mathcal{M}_{n}} T_{n+1}\right\|_{2}^{2} \geq \sum_{\ell, m} \mathbb{E}\left|a_{\ell, m}(n+1)-P_{\mathcal{M}_{\ell, m ; n}} a_{\ell, m}(n+1)\right|^{2} .
$$

The following conditions will be used to state our second main result, with the additional Wold-like decomposition.

Condition 4 Consider the following assumptions:

(i) $\sigma_{\ell}^{2}>0$ for all $\ell \geq 0$;

(ii) $\mathcal{M}_{\ell, m ;-\infty}=\{0\}$ for all $\ell \geq 0, m=-\ell, \ldots, \ell$.

Lemma 1 (Wold Decomposition) An isotropic stationary random field $\{T(x, t),(x, t) \in$ $\mathbb{S}^{2} \times \mathbb{Z}$ \} satisfying Conditions 4 ( $i$ ) can be expressed as

$$
T(x, t)=\sum_{j=0}^{\infty} \Psi_{j} Z(x, t-j)+V(x, t),
$$


in $L^{2}(\Omega)$ and $L^{2}\left(\mathbb{S}^{2} \times \Omega\right)$, where

- $\Psi_{j} Z(x, t-j):=\sum_{\ell, m} \psi_{\ell ; j} a_{\ell, m ; Z}(t-j) Y_{\ell, m}(x)$;

- $\psi_{\ell ; 0}=1$ and $\sum_{j=0}^{\infty}\left|\psi_{\ell ; j}\right|^{2}<\infty$ for all $\ell \geq 0$;

- $a_{\ell, m ; Z}(t) \in \mathcal{M}_{\ell, m ; t}$ for all $\ell, m, t$;

- $Z \sim S W N$ with power spectrum $\left\{\sigma_{\ell}^{2}\right\}$;

- $V(x, t):=\sum_{\ell, m} V_{\ell, m}(t) Y_{\ell, m}(x)$ with $V_{\ell, m}(t) \in \mathcal{M}_{\ell, m ;-\infty}$ for all $\ell, m, t$;

- $\mathbb{E}\left[a_{\ell, m ; Z}(t) \overline{V_{\ell^{\prime}, m^{\prime}}}(s)\right]=0$ for all $\ell, \ell^{\prime}, m, m^{\prime}, t, s$.

Remark 12 Condition $4(i)$ entails that each stationary subprocess $\left\{a_{\ell, m}(t), t \in \mathbb{Z}\right\}$ is nondeterministic [see (Brockwell and Davis 1991, Remark 1 in Sect. 5.7)]. If in addition Condition 4 ( $\mathrm{ii}$ ) holds, the Wold decomposition has no deterministic component $V$ and the subprocesses, as well as the global process, are said to be purely non-deterministic. SPHARMA processes belong to this class.

Remark 13 The $h$-step prediction error $\sigma^{2}(h):=\sum_{\ell, m} \mathbb{E}\left|a_{\ell, m}(t+h)-P_{\mathcal{M}_{\ell, m ; t}} a_{\ell, m}(t+h)\right|^{2}$ is given by

$$
\sigma^{2}(h)=\sum_{\ell}(2 \ell+1) \sigma_{\ell}^{2} \sum_{j=0}^{h-1}\left|\psi_{\ell ; j}\right|^{2} .
$$

For a purely non-deterministic process it is clear that the $h$-step prediction mean squared error converges as $h \rightarrow \infty$ to the total variance of the process.

We are now in the position to present our last main theorem. The statement makes precise the way in which the $L^{2}(\Omega)$ approximations hold.

Theorem 3 An isotropic stationary random field $\left\{T(x, t),(x, t) \in \mathbb{S}^{2} \times \mathbb{Z}\right\}$ satisfying Conditions $4(i)$ and $(i i)$ is such that, for all $\epsilon>0$, there exists integers $L$ and $q$ such that

$$
\mathbb{E}\left|T(x, t)-Z(x, t)-\sum_{j=1}^{q} \Psi_{j ; L} Z(x, t-j)\right|^{2} \leq \epsilon,
$$

where $\Psi_{j ; L} Z(x, t-j):=\sum_{\ell=0}^{L} \sum_{m=-\ell}^{\ell} \psi_{\ell ; j} a_{\ell, m ; Z}(t-j) Y_{\ell, m}(x)$. Moreover, for all $\epsilon>0$, there exist integers $L$ and $p$ such that

$$
\mathbb{E}\left|T(x, t)-\sum_{j=1}^{p} \Phi_{j ; L} T(x, t-j)-Z(x, t)\right|^{2} \leq \epsilon,
$$

where $\Phi_{j ; L} T(x, t-j):=\sum_{\ell=0}^{L} \sum_{m=-\ell}^{\ell} \phi_{\ell ; j} a_{\ell, m}(t) Y_{\ell, m}(x)$ for some coefficients $\left\{\phi_{\ell ; j}\right\}$ such that $\sum_{j=1}^{\infty}\left|\phi_{\ell ; j}\right|^{2}<\infty, \ell=0, \ldots$, L. Both results also hold in the $L^{2}\left(\mathbb{S}^{2} \times \Omega\right)$ sense.

Remark 14 Clearly, it is possible to obtain a $\operatorname{SPHARMA}(p, q)$ approximation, by first applying (17) to $\left\{T(x, t),(x, t) \in \mathbb{S}^{2} \times \mathbb{Z}\right\}$ and then (16) to the residual $\left\{Z(x, t),(x, t) \in \mathbb{S}^{2} \times \mathbb{Z}\right\}$.

\section{Proofs}

Proof (Proposition 1) Define $a_{\ell, m}(t):=\left\langle T_{t}, Y_{\ell, m}\right\rangle_{2}$. For every fixed $(\ell, m)$, we shall prove that $\left\{a_{\ell, m}(t), t \in \mathbb{Z}\right\}$ forms a zero-mean complex-valued stationary sequence, i.e.,

$$
\mathbb{E}\left[a_{\ell, m}(t)\right]=0 ;
$$




$$
\begin{aligned}
& \mathbb{E}\left|a_{\ell, m}(t)\right|^{2}<\infty \\
& \mathbb{E}\left[a_{\ell, m}(t) \overline{a_{\ell, m}}(s)\right]=C_{\ell}(t-s) .
\end{aligned}
$$

Indeed, by Fubini's Theorem we have

$$
\mathbb{E}\left[a_{\ell, m}(t)\right]=\mathbb{E}\left\langle T_{t}, Y_{\ell, m}\right\rangle_{2}=\left\langle 0, Y_{\ell, m}\right\rangle_{2}=0 .
$$

Moreover,

$$
\mathbb{E}\left|a_{\ell, m}(t)\right|^{2} \leq \mathbb{E}\left\|T_{t}\right\|_{2}^{2}<\infty,
$$

and, again by Fubini's Theorem,

$\mathbb{E}\left[a_{\ell, m}(t) \overline{a_{\ell^{\prime}, m^{\prime}}}(s)\right]=\mathbb{E}\left[\left\langle T_{t}, Y_{\ell, m}\right\rangle_{2} \overline{\left\langle T_{s}, Y_{\ell^{\prime}, m^{\prime}}\right\rangle_{2}}\right]=\left\langle\mathscr{R}_{t-s} Y_{\ell, m}, Y_{\ell^{\prime}, m^{\prime}}\right\rangle_{2}=C_{\ell}(t-s) \delta_{\ell}^{\ell^{\prime}} \delta_{m}^{m^{\prime}}$, due to (2).

Proof (Proposition 2) First observe that, for fixed $t \in \mathbb{Z},\left\{T(x, t), x \in \mathbb{S}^{2}\right\}$ is a zero-mean (2-weakly) isotropic spherical random field [see Marinucci and Peccati 2011, Definition 5.9 (2)]. Without loss of generality, take $t=0$ and define the partial sum

$$
T_{L}(x, 0)=\sum_{\ell=0}^{L} \sum_{m=-\ell}^{\ell} a_{\ell, m}(0) Y_{\ell, m}(x), \quad x \in \mathbb{S}^{2},
$$

with $a_{\ell, m}(0)=\int_{\mathbb{S}^{2}} T(x, 0) \overline{Y_{\ell, m}}(x) d x$. Then, from (Marinucci and Peccati 2011, Theorem 5.13), we have that

$$
\lim _{L \rightarrow \infty} \mathbb{E}\left|T(x, 0)-T_{L}(x, 0)\right|^{2}=0,
$$

for every $x \in \mathbb{S}^{2}$. In addition,

$$
\mathbb{E}\left\|T_{0}\right\|_{2}^{2}=\left\|\mathscr{R}_{0}\right\|_{\mathrm{TR}}=\sum_{\ell=0}^{\infty}(2 \ell+1) C_{\ell}(0)<\infty .
$$

Since the limit in (18) is a limit in $L^{2}(\Omega)$, by continuity of inner products, we can write

$$
\langle T(x, 0), T(y, 0)\rangle_{L^{2}(\Omega)}=\lim _{L \rightarrow \infty}\left\langle T_{L}(x, 0), T_{L}(y, 0)\right\rangle_{L^{2}(\Omega)},
$$

for every $(x, y) \in \mathbb{S}^{2} \times \mathbb{S}^{2}$. Consequently,

$$
\begin{aligned}
r_{0}(x, y)=\mathbb{E}[T(x, 0) T(y, 0)] & =\lim _{L \rightarrow \infty} \sum_{\ell=0}^{L} \sum_{m=-\ell}^{\ell} \sum_{\ell^{\prime}=0}^{L} \sum_{m^{\prime}=-\ell^{\prime}}^{\ell^{\prime}} \mathbb{E}\left[a_{\ell, m}(0) \overline{a_{\ell^{\prime}, m^{\prime}}}(0)\right] Y_{\ell, m}(x) \overline{Y_{\ell^{\prime}, m^{\prime}}}(y) \\
& =\sum_{\ell=0}^{\infty} C_{\ell}(0) \sum_{m=-\ell}^{\ell} Y_{\ell, m}(x) \overline{Y_{\ell, m}}(y) \\
& =\sum_{\ell=0}^{\infty} C_{\ell}(0) \frac{2 \ell+1}{4 \pi} P_{\ell}(\langle x, y\rangle),
\end{aligned}
$$

and the limit is pointwise. Now, observe that on the right hand side of this expression we have a sum of continuous functions (Legendre polynomials and $\langle\cdot, \cdot\rangle$ are continuous) that is 
also uniformly convergent. Indeed, for $L>L^{\prime}$,

$$
\sup _{(x, y)}\left|\sum_{\ell=L^{\prime}+1}^{L} C_{\ell}(0) \frac{2 \ell+1}{4 \pi} P_{\ell}(\langle x, y\rangle)\right| \leq \sum_{\ell=L^{\prime}+1}^{\infty} C_{\ell}(0) \frac{2 \ell+1}{4 \pi},
$$

and the sequence of partial sums is Cauchy; here we used the fact that Legendre polynomials are bounded by 1 and (19). Since the space of continuous functions on $\mathbb{S}^{2} \times \mathbb{S}^{2}$ equipped with the sup norm is Banach, this sum must converge to a continuous function, say $\tilde{r}_{0}(\cdot, \cdot)$. However, uniform convergence implies pointwise convergence; hence, by uniqueness of limits, it must be $r_{0}(x, y)=\tilde{r}_{0}(x, y)$, for every $(x, y) \in \mathbb{S}^{2} \times \mathbb{S}^{2}$. Thus, $r_{0}(\cdot, \cdot)$ is continuous.

We can deduce that each $\left\{T(x, t), x \in \mathbb{S}^{2}\right\}, t \in \mathbb{Z}$, is mean-square continuous, i.e., for every $x_{0} \in \mathbb{S}^{2}$,

$$
\lim _{x \rightarrow x_{0}} \mathbb{E}\left|T(x, t)-T\left(x_{0}, t\right)\right|^{2}=0,
$$

by observing that

$$
\mathbb{E}\left|T(x, t)-T\left(x_{0}, t\right)\right|^{2}=r_{0}(x, x)+r_{0}\left(x_{0}, x_{0}\right)-2 r_{0}\left(x, x_{0}\right) .
$$

Continuity of all kernels $r_{t}(\cdot, \cdot), t \in \mathbb{Z}$, follows from (20). Write

$$
r_{t}(x, y)-r_{t}\left(x_{0}, y_{0}\right)=\left(r_{t}(x, y)-r_{t}\left(x_{0}, y\right)\right)+\left(r_{t}\left(x_{0}, y\right)-r_{t}\left(x_{0}, y_{0}\right)\right) .
$$

The Cauchy-Schwarz inequality then gives

$$
\left|r_{t}(x, y)-r_{t}\left(x_{0}, y\right)\right| \leq\left(\mathbb{E}|T(y, 0)|^{2}\right)^{1 / 2}\left(\mathbb{E}\left|T(x, t)-T\left(x_{0}, t\right)\right|^{2}\right)^{1 / 2}
$$

and

$$
\left|r_{t}\left(x_{0}, y\right)-r_{t}\left(x_{0}, y_{0}\right)\right| \leq\left(\mathbb{E}\left|T\left(x_{0}, t\right)\right|^{2}\right)^{1 / 2}\left(\mathbb{E}\left|T(y, 0)-T\left(y_{0}, 0\right)\right|^{2}\right)^{1 / 2},
$$

and the conclusion is immediate.

Lastly, since $r_{t}(\cdot, \cdot)$ is continuous and

$$
\left\|\mathscr{R}_{t}\right\|_{\mathrm{TR}}=\sum_{\ell=0}^{\infty}(2 \ell+1)\left|C_{\ell}(t)\right| \leq \sum_{\ell=0}^{\infty}(2 \ell+1) C_{\ell}(0)=\left\|\mathscr{R}_{0}\right\|_{\mathrm{TR}}<\infty,
$$

due to stationarity, the convergence in (3) must be uniform.

Proof (Proposition 3) The proof follows the same lines of Panaretos and Tavakoli (2013a). Let $\mathbb{H}$ be the Hilbert space of $L^{2}\left(\mathbb{S}^{2} ; \mathbb{C}\right.$ )-valued random elements with finite second moment and $\mathbb{M}_{0}$ be the complex linear space spanned by all finite linear combinations of the $T_{t}$ 's,

$$
\mathbb{M}_{0}:=\left\{\sum_{j=1}^{n} b_{j} T_{j}: n \in \mathbb{N}, b_{j} \in \mathbb{C}, t_{j} \in \mathbb{Z}\right\} \subset \mathbb{H} .
$$

Let $e_{t}: v \mapsto e^{i t v}$, which belongs to the (complex) Hilbert space $L^{2}\left([-\pi, \pi],\left\|\mathscr{F}_{v}\right\|_{\mathrm{TR}} d v\right)$ endowed with the standard inner product

$$
\int_{-\pi}^{\pi} f(\nu) \overline{g(\nu)}\left\|\mathscr{F}_{\nu}\right\|_{\mathrm{TR}} d \nu, \quad f, g \in L^{2}\left([-\pi, \pi],\left\|\mathscr{F}_{\nu}\right\|_{\mathrm{TR}} d \nu\right),
$$


$\left\|\mathscr{F}_{v}\right\|_{\mathrm{TR}}$ being the nuclear norm of the spectral density operator. Now, define the linear operator $E$ by linear extension of the mapping $T_{t} \mapsto e_{t}$. E is well defined and a linear isometry; in particular, the inversion formula (4) gives

$$
\left\langle T_{t}, T_{s}\right\rangle_{\mathbb{H}}=\mathbb{E}\left[\int_{\mathbb{S}^{2}} T(x, t) T(x, s) d x\right]=\int_{-\pi}^{\pi} e^{i(t-s) v}\left\|\mathscr{F}_{\nu}\right\|_{\mathrm{TR}} d \nu .
$$

Then, we extend its domain to $\mathbb{M}$, the closure of $\mathbb{M}_{0}$ in $\mathbb{H}$ (see Panaretos and Tavakoli (2013a) for further details); the extension has a well-defined inverse $E^{-1}: L^{2}\left([-\pi, \pi],\left\|\mathscr{F}_{v}\right\|_{\mathrm{TR}} d \nu\right) \rightarrow$ $\mathbb{M}$. For any $\omega \in(-\pi, \pi]$, we define $Z_{\omega}=E^{-1}\left(\mathbb{1}_{[-\pi, \omega)}\right) \in \mathbb{M}$ and $Z_{-\pi} \equiv 0$. By the isometry property,

$$
\left\langle Z_{\omega}, Z_{\beta}\right\rangle_{\mathbb{H}}=\left\langle E^{-1} \mathbb{1}_{[-\pi, \omega)}, E^{-1} \mathbb{1}_{[-\pi, \beta)}\right\rangle_{\mathbb{H}}=\int_{-\pi}^{\min \{\omega, \beta\}}\left\|\mathscr{F}_{\nu}\right\|_{\mathrm{TR}} d \nu .
$$

Hence, $\omega \mapsto Z_{\omega}$ is an orthogonal increment process.

The proof follows with definition of an operator $\zeta$ as extension of the mapping

$$
\sum_{j=1}^{n} g_{j} \mathbb{1}_{\left[\omega_{j}, \omega_{j+1}\right)} \mapsto \sum_{j=1}^{n} g_{j}\left(Z_{\omega_{j+1}}-Z_{\omega_{j}}\right)
$$

The operator $\zeta$ is, by (21), an isomorphism with domain $L^{2}\left([-\pi, \pi],\left\|\mathscr{F}_{\nu}\right\|_{\mathrm{TR}} d \nu\right)$, and in addition $\zeta=E^{-1}$. This in turn implies $T_{t}=E^{-1}\left(e_{t}\right)=\zeta\left(e_{t}\right)$. If $g$ is cadlag with a finite number of jumps, then $\zeta(g)$ is in fact the Riemann-Stieltjes integral (in the mean square sense) with respect to the orthogonal increment process $Z_{\omega}$ :

$$
\zeta(g)=\int_{-\pi}^{\pi} g(\lambda) d Z_{\lambda} .
$$

In conclusion, $T_{t}=\int_{-\pi}^{\pi} e^{i t \lambda} d Z_{\lambda}$, as claimed.

The next proof does not go through the eigenvalue-eigenfunction decomposition of $\mathscr{F}_{\lambda}$ [see Panaretos and Tavakoli (2013a)], but is directly based on the standard orthonormal basis of spherical harmonics.

Proof (Proposition 4) Recall from Proposition 1 that, for every fixed $(\ell, m),\left\{a_{\ell, m}(t), t \in \mathbb{Z}\right\}$ is a zero-mean complex-valued stationary sequence and

$$
\mathbb{E}\left[a_{\ell, m}(t) \overline{a_{\ell^{\prime}, m^{\prime}}}(s)\right]=C_{\ell}(t-s) \delta_{\ell}^{\ell^{\prime}} \delta_{m}^{m^{\prime}} .
$$

Therefore, as a result of the Spectral Theorem for stationary time series [see for instance Brockwell and Davis (1991)], the following representation holds

$$
a_{\ell, m}(t)=\int_{-\pi}^{\pi} e^{i \lambda t} d \alpha_{\ell, m}(\lambda), \quad \text { a.s., }
$$

where $\left\{\alpha_{\ell, m}(\lambda),-\pi \leq \lambda \leq \pi\right\}$ is an orthogonal increment process, and the stochastic integral involved can be understood as a Riemann-Stieltjes limit, in the sense that

$$
\mathbb{E}\left|a_{\ell, m}(t)-\sum_{j=1}^{J} e^{i \lambda_{j} t}\left[\alpha_{\ell, m}\left(\lambda_{j+1}\right)-\alpha_{\ell, m}\left(\lambda_{j}\right)\right]\right|^{2} \rightarrow 0, \quad J \rightarrow \infty,
$$

where $-\pi=\lambda_{1}<\cdots<\lambda_{J+1}=\pi$ and $\max _{j=1, \ldots, J}\left|\lambda_{j+1}-\lambda_{j}\right| \rightarrow 0$ as $J \rightarrow \infty$ 
Moreover, recall from the Spectral Theorem for isotropic random fields on $\mathbb{S}^{2}$ that

$$
\mathbb{E}\left|T(x, t)-\sum_{\ell=0}^{L} \sum_{m=-\ell}^{\ell} \int_{-\pi}^{\pi} e^{i \lambda t} d \alpha_{\ell, m}(\lambda) Y_{\ell, m}\right|^{2} \rightarrow 0, \quad L \rightarrow \infty .
$$

Now we prove that $\alpha_{\ell, m}(\lambda) \stackrel{\text { a.s. }}{=}\left\langle Z_{\lambda}, Y_{\ell, m}\right\rangle_{2}, Z_{\lambda}$ as defined in Proposition 3

For a fixed $\lambda, \alpha_{\ell, m}(\lambda) \in \overline{\operatorname{span}\left\{a_{\ell, m}(t), t \in \mathbb{Z}\right\}}=\overline{\operatorname{span}\left\{\left\langle T_{t}, Y_{\ell, m}\right\rangle_{2}, t \in \mathbb{Z}\right\}} \subset L^{2}(\Omega)$. Indeed, from Brockwell and Davis (1991), we know that there exist a sequence $\left\{\alpha_{j}\right\}_{j \in \mathbb{Z}} \subset \mathbb{C}$ such that

$$
\mathbb{E}\left|\alpha_{\ell, m}(\lambda)-\sum_{|j| \leq k} \alpha_{j}\left\langle T_{t_{j}}, Y_{\ell, m}\right\rangle_{2}\right|^{2} \rightarrow 0, \quad k \rightarrow \infty .
$$

The sequence is given by

$$
\alpha_{j}=\frac{1}{2 \pi} \int_{-\pi}^{\pi} \mathbb{1}_{[-\pi, \lambda)}(\nu) e^{-i j v} d \nu, \quad j \in \mathbb{Z} .
$$

Now,

$$
\begin{aligned}
\mathbb{E}\left|\left\langle Z_{\lambda}, Y_{\ell, m}\right\rangle_{2}-\sum_{|j| \leq k} \alpha_{j}\left\langle T_{t_{j}}, Y_{\ell, m}\right\rangle_{2}\right|^{2} & =\mathbb{E}\left|\left\langle Z_{\lambda}-\sum_{|j| \leq k} \alpha_{j} T_{t_{j}}, Y_{\ell, m}\right\rangle_{2}\right|^{2} \\
& \leq \mathbb{E}\left\|Z_{\lambda}-\sum_{|j| \leq k} \alpha_{j} T_{t_{j}}\right\|_{L^{2}\left(\mathbb{S}^{2} ; \mathbb{C}\right)}^{2}
\end{aligned}
$$

by Cauchy-Schwarz inequality e orthonormality of the $Y_{\ell, m}$ 's.

We just need to prove that

$$
\mathbb{E}\left\|Z_{\lambda}-\sum_{|j| \leq k} \alpha_{j} T_{t_{j}}\right\|_{L^{2}\left(\mathbb{S}^{2} ; \mathbb{C}\right)}^{2} \rightarrow 0, \quad k \rightarrow \infty .
$$

Recall that $\left\{\alpha_{j}, j \in \mathbb{Z}\right\}$ as defined in (22) represent the Fourier coefficients of the indicator function $\mathbb{1}_{[-\pi, \lambda)}(\cdot)$. Then, its $k$-th order Fourier series approximation is given by

$$
h_{k}(\cdot)=\sum_{|j| \leq k} a_{j} e^{i k}
$$

and $\sum_{|j| \leq k} \alpha_{j} T_{t_{j}}=E^{-1}\left(h_{k}\right)$, where $E$ is the isomorphism of Proposition 5. Since $\left\|\mathscr{F}_{\lambda}\right\|_{\mathrm{TR}}$ is bounded uniformly over $\lambda$ by assumption, it holds that

$$
\int_{-\pi}^{\pi}\left|h_{k}(v)-\mathbb{1}_{[-\pi, \lambda)}(v)\right|^{2}\left\|\mathscr{F}_{\nu}\right\|_{\mathrm{TR}} d v \rightarrow 0, \quad k \rightarrow \infty .
$$

By continuity of $E$, we conclude that

$$
E^{-1}\left(h_{k}\right) \rightarrow E^{-1}\left(\mathbb{1}_{[-\pi, \lambda)}\right)=Z_{\lambda}, \quad k \rightarrow \infty,
$$

in the $L^{2}$-sense. 
Moreover, see Panaretos and Tavakoli (2013a),

$$
\mathbb{E}\left[Z_{\omega}(x) \overline{Z_{\beta}(y)}\right]=\int_{-\pi}^{\min (\omega, \beta)} f_{\alpha}(x, y) d \alpha, \quad \text { a.e.. }
$$

Under isotropy this is equal to

$$
\mathbb{E}\left[Z_{\omega}(x) \overline{Z_{\beta}(y)}\right]=\sum_{\ell=0}^{\infty} \frac{2 \ell+1}{4 \pi} \int_{-\pi}^{\min (\omega, \beta)} f_{\ell}(\alpha) d \alpha P_{\ell}(\langle x, y\rangle), \quad \text { a.e., }
$$

which means that

$$
\mathbb{E}\left[\alpha_{\ell, m}(\omega) \alpha_{\ell^{\prime}, m^{\prime}}(\beta)\right]=\int_{-\pi}^{\min (\omega, \beta)} f_{\ell}(\alpha) d \alpha \delta_{\ell}^{\ell^{\prime}} \delta_{m}^{m^{\prime}} .
$$

Note that

$$
0 \leq \int_{-\pi}^{\min (\omega, \beta)} f_{\ell}(\alpha) d \alpha \leq \int_{-\pi}^{\pi} f_{\ell}(\alpha) d \alpha=C_{\ell}(0)
$$

Proof (Proposition 5) In the first part of the proof we show that there exists a unique isotropic stationary solution to (8) and it is given by (14). This is done in two steps. First we prove that the $T(x, t)=\lim _{k \rightarrow \infty} T_{k}(x, t)$ is a solution of the $\operatorname{SPHARMA}(p, q)$ Eq. (8); and then we prove that any isotropic stationary solution of (8) takes the form (14).

First note that, under Condition 2, for any $\ell \geq 0, \theta_{\ell}(z) / \phi_{\ell}(z)$ has a power series expansion, that is,

$$
\theta_{\ell}(z) / \phi_{\ell}(z)=\sum_{j=0}^{\infty} \psi_{\ell ; j} z^{j}=\psi_{\ell}(z), \quad|z| \leq 1,
$$

and

$$
\sum_{j=0}^{\infty}\left|\psi_{\ell ; j}\right|<\infty
$$

see (Brockwell and Davis 1991, Proof of Theorem 3.1.1, p. 85).

Now, let us show first that the sequence $\left\{T_{k}\right\}$ is Cauchy. Indeed we have, for $k^{\prime}>k, L_{k^{\prime}}>$ $L_{k}$,

$$
\begin{aligned}
T_{k^{\prime}}(x, t)-T_{k}(x, t) & =\sum_{\ell=0}^{L_{k}} \sum_{m=-\ell}^{\ell} \sum_{j=k+1}^{k^{\prime}} \psi_{\ell ; j} a_{\ell, m ; Z}(t-j) Y_{\ell, m}(x) \\
& +\sum_{\ell=L_{k}+1}^{L_{k^{\prime}}} \sum_{m=-\ell}^{\ell} \sum_{j=0}^{k^{\prime}} \psi_{\ell ; j} a_{\ell, m ; Z}(t-j) Y_{\ell, m}(x)
\end{aligned}
$$

and, therefore,

$$
\begin{aligned}
& \mathbb{E}\left|T_{k^{\prime}}(x, t)-T_{k}(x, t)\right|^{2} \\
= & \sum_{\ell=0}^{L_{k}} \frac{2 \ell+1}{4 \pi} C_{\ell ; Z} \sum_{j=k+1}^{k^{\prime}}\left|\psi_{\ell ; j}\right|^{2}+\sum_{\ell=L_{k}+1}^{L_{k^{\prime}}} \frac{2 \ell+1}{4 \pi} C_{\ell ; Z} \sum_{j=0}^{k^{\prime}}\left|\psi_{j ; \ell}\right|^{2}
\end{aligned}
$$




$$
\leq \sum_{\ell=0}^{\infty} \frac{2 \ell+1}{4 \pi} C_{\ell ; Z} \sum_{j=k+1}^{\infty}\left|\psi_{\ell ; j}\right|^{2}+\sum_{\ell=L_{k}+1}^{\infty} \frac{2 \ell+1}{4 \pi} C_{\ell ; Z} \sum_{j=0}^{\infty}\left|\psi_{j ; \ell}\right|^{2} .
$$

For $\ell \geq 0$, consider the stationary process

$$
X_{\ell}(t)=\sum_{j=0}^{\infty} \psi_{\ell ; j} \varepsilon(t-j), \quad t \in \mathbb{Z}
$$

here we take $\{\varepsilon(t), t \in \mathbb{Z}\}$ to be a white noise sequence with variance identically equal to one. The spectral density of $\left\{X_{\ell}(t), t \in \mathbb{Z}\right\}$ is given by [see Brockwell and Davis (1991)]

$$
g_{\ell}(\lambda):=\frac{1}{2 \pi}\left|\sum_{j=0}^{\infty} \psi_{j ; \ell} e^{i \lambda j}\right|^{2}=\frac{1}{2 \pi}\left|\psi_{\ell}\left(e^{i \lambda}\right)\right|^{2}=\frac{1}{2 \pi}\left|\frac{\theta_{\ell}\left(e^{i \lambda}\right)}{\phi_{\ell}\left(e^{i \lambda}\right)}\right|^{2}, \quad \lambda \in[-\pi, \pi] .
$$

Now, recall the identity

$$
\mathbb{V}\left[X_{\ell}(t)\right]=\sum_{j=0}^{\infty}\left|\psi_{\ell ; j}\right|^{2}=\int_{-\pi}^{\pi} g_{\ell}(\lambda) d \lambda
$$

whence

$$
\sum_{j=0}^{\infty}\left|\psi_{\ell ; j}\right|^{2}=\frac{1}{2 \pi} \int_{-\pi}^{\pi}\left|\psi_{\ell}\left(e^{i \lambda}\right)\right|^{2} d \lambda=\frac{1}{2 \pi} \int_{-\pi}^{\pi}\left|\frac{\theta_{\ell}\left(e^{i \lambda}\right)}{\phi_{\ell}\left(e^{i \lambda}\right)}\right|^{2} d \lambda .
$$

On the one hand, we have

$$
\left|\theta_{\ell}\left(e^{i \lambda}\right)\right| \leq 1+\sum_{j=1}^{q} \theta_{\ell ; j}
$$

which is bounded uniformly over $\ell$, since $\theta_{\ell ; j} \rightarrow 0, \ell \rightarrow \infty, j=1, \ldots, q$. On the other hand, under Condition 2, for the non-degenerate polynomials it holds that

$$
\left|\phi_{\ell}\left(e^{i \lambda}\right)\right|=\prod_{u=1}^{r_{\ell}}\left|1-\xi_{\ell ; u}^{-1} e^{i \lambda}\right|^{s_{\ell ; u}} \geq \prod_{u=1}^{r_{\ell}}\left(1-\left|\xi_{\ell ; u}^{-1}\right|\right)^{s_{\ell ; u}} \geq\left(1-\xi_{*}^{-1}\right)^{p}>0,
$$

see Remark 8. Consequently,

$$
\sum_{j=0}^{\infty}\left|\psi_{\ell ; j}\right|^{2}=\frac{1}{2 \pi} \int_{-\pi}^{\pi}\left|\frac{\theta_{\ell}\left(e^{i \lambda}\right)}{\phi_{\ell}\left(e^{i \lambda}\right)}\right|^{2} d \lambda \leq M_{1}
$$

where $M_{1}$ is a positive constant not depending on $\ell$, and

$$
\sum_{\ell=0}^{\infty} \sum_{j=0}^{\infty}\left|\psi_{\ell ; j}\right|^{2} \frac{2 \ell+1}{4 \pi} C_{\ell ; Z}<\infty .
$$

Then, by the Dominated Convergence Theorem, we have

$$
\lim _{k \rightarrow \infty} \sum_{\ell=0}^{\infty} \sum_{j=k+1}^{\infty}\left|\psi_{j ; \ell}\right|^{2} \frac{2 \ell+1}{4 \pi} C_{\ell ; Z}=\sum_{\ell=0}^{\infty}\left\{\lim _{k \rightarrow \infty} \sum_{j=k+1}^{\infty}\left|\psi_{j ; \ell}\right|^{2}\right\} \frac{2 \ell+1}{4 \pi} C_{\ell ; Z}=0
$$


and (23) $\rightarrow 0$ as $k \rightarrow \infty$, so that $\left\{T_{k}\right\}$ is indeed a Cauchy sequence. The proof that it satisfies (8) is standard; without loss of generality here we consider the $\operatorname{SPHAR}(p)$ case. Hence, we have that

$$
\begin{aligned}
& \mathbb{E}\left|T(x, t)-\sum_{j=1}^{p}\left(\Phi_{j} T(\cdot, t-j)\right)(x)-Z(x, t)\right|^{2} \\
= & \lim _{k \rightarrow \infty} \mathbb{E}\left|T_{k}(x, t)-\sum_{j=1}^{p}\left(\Phi_{j} T_{k}(\cdot, t-j)\right)(x)-Z(x, t)\right|^{2} \\
= & \lim _{k \rightarrow \infty} \mathbb{E}\left|\sum_{j=1}^{p} \sum_{\ell=0}^{L_{k}} \sum_{m=-\ell}^{\ell} \sum_{h=k-j+1}^{k} \phi_{\ell ; j} \psi_{h ; \ell} a_{\ell, m ; Z}(t-j-h) Y_{\ell, m}(x)\right|^{2} \\
\leq & \sum_{j=1}^{p} \lim _{k \rightarrow \infty} \mathbb{E}\left|\sum_{\ell=0}^{L_{k}} \sum_{m=-\ell}^{\ell} \sum_{h=k-j+1}^{k} \phi_{\ell ; j} \psi_{h ; \ell} a_{\ell, m ; Z}(t-j-h) Y_{\ell, m}(x)\right|^{2},
\end{aligned}
$$

which again is easily shown to be zero by $\lim _{k \rightarrow \infty} \sum_{h=k-j+1}^{\infty}\left|\psi_{h ; \ell}\right|^{2}=0$ and Dominated Convergence Theorem. The argument involving the $L^{2}\left(\mathbb{S}^{2} \times \Omega\right)$ limit is analogous.

To complete the first part of the proof, we need to show that if $\left\{U(x, t),(x, t) \in \mathbb{S}^{2} \times \mathbb{Z}\right\}$ is an isotropic stationary solution of (8), then we must have

$$
U(x, t)=\sum_{\ell=0}^{\infty} \sum_{m=-\ell}^{\ell} \sum_{j=0}^{\infty} \psi_{j ; \ell} a_{\ell, m ; Z}(t-j) Y_{\ell, m}(x)
$$

in $L^{2}\left(\mathbb{S}^{2} \times \Omega\right)$ and $L^{2}(\Omega)$. If $\left\{U(x, t),(x, t) \in \mathbb{S}^{2} \times \mathbb{Z}\right\}$ is an isotropic stationary solution of (8), then $a_{\ell, m ; U}(t)=\int_{\mathbb{S}^{2}} U(x, t) Y_{\ell, m}(x) d x$ is a stationary solution of the $\operatorname{ARMA}(p, q)$ Eq. (9) and, under Condition 2,

$$
a_{\ell, m ; U}(t)=\sum_{j=0}^{\infty} \psi_{\ell ; j} a_{\ell, m ; Z}(t-j), \quad \text { in } L^{2}(\Omega),
$$

with $\mathbb{E}\left|a_{\ell, m ; U}(t)\right|^{2}=C_{\ell ; Z} \sum_{j=0}^{\infty}\left|\psi_{\ell ; j}\right|^{2}$, due to (Brockwell and Davis 1991, Theorem 3.1.1). Thus, we have

$$
\begin{aligned}
& \mathbb{E}\left\|\sum_{\ell=0}^{L_{k}} \sum_{m=-\ell}^{\ell} a_{\ell, m ; U}(t) Y_{\ell, m}-\sum_{\ell=0}^{L_{k}} \sum_{m=-\ell}^{\ell} \sum_{j=0}^{k} \psi_{\ell ; j} a_{\ell, m ; Z}(t-j) Y_{\ell, m}\right\|_{L^{2}\left(\mathbb{S}^{2} ; \mathbb{C}\right)}^{2} \\
= & \sum_{\ell=0}^{L_{k}}(2 \ell+1) C_{\ell ; Z} \sum_{j=k+1}^{\infty}\left|\psi_{\ell ; j}\right|^{2},
\end{aligned}
$$

which goes to zero as $k \rightarrow \infty$. Hence, by triangular inequality,

$$
\mathbb{E}\left\|U(\cdot, t)-\sum_{\ell=0}^{L_{k}} \sum_{m=-\ell}^{\ell} \sum_{j=0}^{k} \psi_{\ell ; j} a_{\ell, m ; Z}(t-j) Y_{\ell, m}\right\|_{L^{2}\left(\mathbb{S}^{2} ; \mathbb{C}\right)}^{2} \rightarrow 0, \quad k \rightarrow \infty .
$$


The same result holds in the sense of convergence in $L^{2}(\Omega)$, for every fixed pair $(x, t)$. Indeed, we have

$$
\begin{aligned}
& \mathbb{E}\left|\sum_{\ell=0}^{L_{k}} \sum_{m=-\ell}^{\ell} a_{\ell, m ; U}(t) Y_{\ell, m}(x)-\sum_{\ell=0}^{L_{k}} \sum_{m=-\ell}^{\ell} \sum_{j=0}^{k} \psi_{\ell ; j} a_{\ell, m ; Z}(t-j) Y_{\ell, m}(x)\right|^{2} \\
= & \sum_{\ell=0}^{L_{k}} \frac{2 \ell+1}{4 \pi} C_{\ell ; Z} \sum_{j=k+1}^{\infty}\left|\psi_{\ell ; j}\right|^{2} .
\end{aligned}
$$

Lastly, define

$$
f_{\ell}(\lambda):=C_{\ell ; Z} \cdot g_{\ell}(\lambda)=\frac{C_{\ell ; Z}}{2 \pi}\left|\frac{\theta_{\ell}\left(e^{i \lambda}\right)}{\phi_{\ell}\left(e^{i \lambda}\right)}\right|^{2}, \quad \lambda \in[-\pi, \pi],
$$

and observe that, by (24) and (25),

$$
\sum_{\ell=0}^{\infty}(2 \ell+1) f_{\ell}(\lambda) \leq M_{2} \sum_{\ell=0}^{\infty}(2 \ell+1) C_{\ell ; Z}<\infty,
$$

where $M_{2}$ is a positive constant not depending on $\ell$ and $\lambda$. Thus, the spectral density kernel at frequency $\lambda \in[-\pi, \pi]$ is well defined as the limit in $\|\cdot\|_{\infty}$

$$
f_{\lambda}(x, y):=\sum_{\ell=0}^{\infty} f_{\ell}(\lambda) \frac{2 \ell+1}{4 \pi} P_{\ell}(\langle x, y\rangle),
$$

and $\mathscr{F}_{\lambda}$ is the corresponding operator induced through right-integration with uniformly bounded $\left\|\mathscr{F}_{\lambda}\right\|_{\mathrm{TR}}$. Moreover,

$$
\begin{aligned}
\left\|\mathscr{F}_{\lambda_{1}}-\mathscr{F}_{\lambda_{2}}\right\|_{\mathrm{TR}} & =\sum_{\ell=0}^{\infty}(2 \ell+1)\left|f_{\ell}\left(\lambda_{1}\right)-f_{\ell}\left(\lambda_{2}\right)\right| \\
& \leq C \sum_{\ell=0}^{L}\left|g_{\ell}\left(\lambda_{1}\right)-g_{\ell}\left(\lambda_{2}\right)\right|+2 M_{2} \sum_{\ell>L}(2 \ell+1) C_{\ell ; Z},
\end{aligned}
$$

where $C=\sup _{\ell \geq 0}(2 \ell+1) C_{\ell ; Z}$. Fixing $\epsilon>0$, we can choose $L=L(\epsilon)>0$ such that

$$
2 M_{2} \sum_{\ell>L}(2 \ell+1) C_{\ell ; Z} \leq \epsilon / 2 .
$$

Now, under Condition 2, the functions $\lambda \mapsto g_{\ell}(\lambda), \ell \geq 0$, are uniformly continuous in $[-\pi, \pi]$; hence, we can choose $\delta=\delta(L(\epsilon), \epsilon)=\delta(\epsilon)>0$ such that

$$
C \sum_{\ell=0}^{L}\left|g_{\ell}\left(\lambda_{1}\right)-g_{\ell}\left(\lambda_{2}\right)\right| \leq \epsilon / 2 .
$$

Thus, uniform continuity follows.

Proof (Theorem 1) First observe that, under Condition $1(i), \forall \epsilon>0$ there exists $L$ such that

$$
\sup _{\lambda \in[-\pi, \pi]}\left(\sum_{\ell>L}(2 \ell+1)\left|f_{\ell}(\lambda)\right|^{2}\right)^{1 / 2} \leq \epsilon / 2 .
$$


Indeed, $\left\|f_{\lambda}\right\|_{2} \leq \sum_{t \in \mathbb{Z}}\left\|r_{t}\right\|_{2}$, then

$$
\begin{aligned}
\left(\sum_{\ell>L}(2 \ell+1)\left|f_{\ell}(\lambda)\right|^{2}\right)^{1 / 2} & \leq \sum_{t=-\infty}^{\infty}\left(\sum_{\ell>L}(2 \ell+1)\left|C_{\ell}(t)\right|^{2}\right)^{1 / 2} \\
& \leq \sum_{t=-\infty}^{\infty}\left(\sum_{\ell=0}^{\infty}(2 \ell+1)\left|C_{\ell}(t)\right|^{2}\right)^{1 / 2}<\infty .
\end{aligned}
$$

From (Brockwell and Davis 1991, Theorem 4.4.3 and Corollary 4.4.1), take $\frac{\epsilon}{2(L+1)^{2}}$, then there exists an $\operatorname{MA}\left(q_{\ell}\right)$ process with white noise variance $\sigma_{\ell}^{2}=\left(1+\theta_{\ell ; 1}^{2}+\cdots+\right.$ $\left.\theta_{\ell ; q}^{2}\right)^{-1} \int_{-\pi}^{\pi} f_{\ell}(\lambda) d \lambda$ and spectral density $\tilde{f}_{\ell}$ such that

$$
\left|\tilde{f}_{\ell}(\lambda)-f_{\ell}(\lambda)\right| \leq \frac{\epsilon}{2(L+1)^{2}}, \quad \text { for all } \lambda \in[-\pi, \pi] .
$$

Now, define

$$
\tilde{f}_{\lambda}(\langle x, y\rangle)=\sum_{\ell=0}^{L} \tilde{f}_{\ell}(\lambda) \frac{2 \ell+1}{4 \pi} P_{\ell}(\langle x, y\rangle),
$$

and $\tilde{\mathscr{F}}_{\lambda}$ the operator induced by right integration. Take $q=\max _{\ell \leq L} q_{\ell}$. Then, we have

$$
\left\|\tilde{f}_{\lambda}-f_{\lambda}\right\|_{2} \leq\left(\sum_{\ell=0}^{L}(2 \ell+1)\left|\tilde{f}_{\ell}(\lambda)-f_{\ell}(\lambda)\right|^{2}\right)^{1 / 2}+\left(\sum_{\ell>L}(2 \ell+1)\left|f_{\ell}(\lambda)\right|^{2}\right)^{1 / 2} \leq \epsilon,
$$

uniformly over $\lambda \in[-\pi, \pi]$.

Similarly, under Condition 1 ( $i$ i $)$,

$$
\left\|\tilde{\mathscr{F}}_{\lambda}-\mathscr{F}_{\lambda}\right\|_{\mathrm{TR}} \leq \sum_{\ell=0}^{L}(2 \ell+1)\left|\tilde{f}_{\ell}(\lambda)-f_{\ell}(\lambda)\right|+\sum_{\ell>L}(2 \ell+1) f_{\ell}(\lambda) \leq \epsilon,
$$

for all $\lambda \in[-\pi, \pi]$.

Proof (Theorem 2) This proof follows the same lines of the previous one. Here, we make use of (Brockwell and Davis 1991, Theorem 4.4.3 and Corollary 4.4.2), which ensure that for $\ell=0, \ldots, L$ there exists an $\operatorname{AR}\left(p_{\ell}\right)$ process with spectral density $\tilde{f}_{\ell}$ such that

$$
\left|\tilde{f}_{\ell}(\lambda)-f_{\ell}(\lambda)\right| \leq \frac{\epsilon}{2(L+1)^{2}}, \quad \text { for all } \lambda \in[-\pi, \pi] .
$$

We then take $p=\max _{\ell \leq L} p_{\ell}$.

Proof (Lemma 1) We just prove convergence in the $L^{2}(\Omega)$ sense; the $L^{2}\left(\mathbb{S}^{2} \times \Omega\right)$ argument is analogous. First of all, by Theorem 5.7.1 in Brockwell and Davis (1991), we can deduce that, for fixed $(\ell, m)$,

$$
a_{\ell, m}(t)=\sum_{j=0}^{\infty} \psi_{\ell ; j} a_{\ell, m ; Z}(t-j)+V_{\ell, m}(t), \quad \text { in } L^{2}(\Omega),
$$

and

$$
\infty>\sum_{\ell}(2 \ell+1) C_{\ell}(0)=\sum_{\ell}(2 \ell+1) \sigma_{\ell}^{2} \sum_{j=0}^{\infty}\left|\psi_{\ell ; j}\right|^{2}+\sum_{\ell}(2 \ell+1) v_{\ell}
$$


were $v_{\ell}=\mathbb{E}\left|V_{\ell, m}(t)\right|^{2}$. In addition, since $a_{\ell, m ; Z}(t) \in \mathcal{M}_{\ell, m ; t}$ and $V_{\ell, m}(t) \in \mathcal{M}_{\ell, m ;-\infty}$, it is clear that $\mathbb{E}\left[a_{\ell, m ; Z}(t) \overline{a_{\ell^{\prime}, m^{\prime} ; Z}}(s)\right]=\sigma_{\ell}^{2} \delta_{t}^{s} \delta_{\ell}^{\ell^{\prime}} \delta_{m}^{m^{\prime}}$, and $\mathbb{E}\left[a_{\ell, m ; Z}(t) \overline{V_{\ell^{\prime}, m^{\prime}}}(s)\right]=0$ for all $\ell, \ell^{\prime}, m, m^{\prime}, t, s$.

Hence, the sequence $\sum_{\ell=0}^{L} \sum_{m=-\ell}^{\ell} \psi_{\ell ; j} a_{\ell, m ; Z}(t-j) Y_{\ell, m}(x)$ is Cauchy in $L^{2}(\Omega)$ since, for $L>L^{\prime}$,

$$
\mathbb{E}\left|\sum_{\ell=L^{\prime}+1}^{L} \sum_{m=-\ell}^{\ell} \psi_{\ell ; j} a_{\ell, m ; Z}(t-j) Y_{\ell, m}(x)\right|^{2}=\sum_{\ell=L^{\prime}+1}^{L}\left|\psi_{\ell ; j}\right|^{2} \frac{2 \ell+1}{4 \pi} \sigma_{\ell}^{2} \rightarrow 0
$$

and $\Psi_{j} Z(x, t-j)$ is well defined. Similarly $Z(x, t):=\sum_{\ell, m} a_{\ell, m ; Z}(t) Y_{\ell, m}(x)$ and $V(x, t)$ are also well defined. Moreover, $\sum_{j} \Psi_{j} Z(x, t-j)$ is Cauchy. Indeed, for $J>J^{\prime}$,

$$
\begin{aligned}
\mathbb{E}\left|\sum_{j=J^{\prime}+1}^{J} \Psi_{j} Z(x, t-j)\right|^{2} & =\lim _{L \rightarrow \infty} \mathbb{E}\left|\sum_{j=J^{\prime}+1}^{J} \sum_{\ell=0}^{L} \sum_{m=-\ell}^{\ell} \psi_{\ell ; j} a_{\ell, m ; Z}(t-j) Y_{\ell, m}(x)\right|^{2} \\
& =\sum_{j=J^{\prime}+1}^{J} \sum_{\ell=0}^{\infty}\left|\psi_{\ell ; j}\right|^{2} \frac{2 \ell+1}{4 \pi} \sigma_{\ell}^{2} \rightarrow 0 .
\end{aligned}
$$

Then, we have

$$
\begin{aligned}
& \mathbb{E}\left|T(x, t)-\sum_{j=0}^{J} \Psi_{j} Z(x, t-j)-V(x, t)\right|^{2} \\
= & \lim _{L \rightarrow \infty} \mathbb{E}\left|\sum_{\ell=0}^{L} \sum_{m=-\ell}^{\ell}\left(a_{\ell, m}(t)-\sum_{j=0}^{J} \psi_{\ell ; j} a_{\ell, m ; Z}(t-j)-V_{\ell, m}(t)\right) Y_{\ell, m}(x)\right|^{2} \\
= & \sum_{\ell=0}^{\infty} \frac{2 \ell+1}{4 \pi} \sigma_{\ell}^{2} \sum_{j=J+1}^{\infty}\left|\psi_{\ell ; j}\right|^{2},
\end{aligned}
$$

and by Dominated Convergence Theorem we conclude the proof.

Proof (Theorem 3) Under Condition 4 (ii), the Wold decomposition has no deterministic component, that is, $V(x, t)=0$ for all $(x, t) \in \mathbb{S}^{2} \times \mathbb{Z}$. Then, as a consequence of Lemma 1 , for all $\epsilon>0$, there exists integers $L$ and $q$ such that

$$
\mathbb{E}\left|T(x, t)-Z(x, t)-\sum_{j=1}^{q} \Psi_{j ; L} Z(x, t-j)\right|^{2} \leq \epsilon .
$$

Moreover, since $a_{\ell, m ; Z}(t) \in \mathcal{M}_{\ell, m ; t}$ for all $\ell, m, t$, the representation can be inverted:

$$
a_{\ell, m}(t)=\sum_{j=1}^{\infty} \phi_{\ell ; j} a_{\ell, m}(t-j)+a_{\ell, m ; Z}(t),
$$

with $\sum_{j=1}^{\infty}\left|\phi_{\ell ; j}\right|^{2}<\infty$. Then, for all $\epsilon>0$, there exist integers $L$ and $p$ such that

$$
\mathbb{E}\left|T(x, t)-\sum_{j=1}^{p} \Phi_{j ; L} T(x, t-j)-Z(x, t)\right|^{2} \leq \epsilon .
$$


Similarly, it is possible to show the $L^{2}\left(\mathbb{S}^{2} \times \Omega\right)$ approximation.

Acknowledgements The research was supported by MIUR Excellence Department Project awarded to the Department of Mathematics, University of Rome Tor Vergata, CUP E83C18000100006. The author is also grateful to Domenico Marinucci for many insightful discussions and suggestions.

Funding Open Access funding provided by EPFL Lausanne.

\section{Declarations}

Conflict of interest The author declare that there is no conflict of interest.

Open Access This article is licensed under a Creative Commons Attribution 4.0 International License, which permits use, sharing, adaptation, distribution and reproduction in any medium or format, as long as you give appropriate credit to the original author(s) and the source, provide a link to the Creative Commons licence, and indicate if changes were made. The images or other third party material in this article are included in the article's Creative Commons licence, unless indicated otherwise in a credit line to the material. If material is not included in the article's Creative Commons licence and your intended use is not permitted by statutory regulation or exceeds the permitted use, you will need to obtain permission directly from the copyright holder. To view a copy of this licence, visit http://creativecommons.org/licenses/by/4.0/.

\section{References}

Aue A, van Delft A (2020) Testing for stationarity of functional time series in the frequency domain. Ann Stat 48(5):2505-2547

Berg C, Porcu E (2017) From Schoenberg coefficients to Schoenberg functions. Constr Approx 45:217-241

Bosq D (2000) Linear processes in function spaces: theory and applications. Springer, Berlin

Bosq D (2002) Estimation of mean and covariance operator of autoregressive processes in Banach spaces. Stat Inference Stoch Processes 5:287-306

Bosq D, Blanke D (2007) Inference and prediction in large dimensions. Wiley, New Jersey

Brockwell PJ, Davis RA (1991) Time series: theory and methods, 2nd edn. Springer Series in Statistics. Springer, Berlin

Caponera A, Durastanti C, Vidotto A (2021) LASSO estimation for spherical autoregressive processes. Stoch Process Appl 137:167-199

Caponera A, Marinucci D (2021) Asymptotics for spherical functional autoregressions. Ann Stat 49(1):346369

Castruccio S (2016) Assessing the spatio-temporal structure of annual and seasonal surface temperature for CMIP5 and reanalysis. Spat Stat 18:179-193

Castruccio S, Stein ML (2013) Global space-time models for climate ensembles. Ann Appl Stat 7(3):15931611

De la Cerda J, Clarke Alegría A, Porcu E (2018) Regularity properties and simulations of Gaussian random fields on the sphere cross time. Electron J Stat 12:399-426

Christakos G (2005) Random field models in earth sciences. Elsevier, Amsterdam

Dehling H, Sharipov O (2005) Estimation of mean and covariance operator for Banach space valued autoregressive processes with dependent innovations. Stat Inference Stoch Process 8:137-149

Gneiting T (2002) Nonseparable, stationary covariance functions for space-time data. J Am Stat Assoc 97:590600

Gneiting T (2013) Strictly and non-strictly positive definite functions on spheres. Bernoulli 19(4):1327-1349

Hörmann S, Kokoszka P, Nisol G (2018) Testing for periodicity in functional time series. Ann Stat 46(6A):2960-2984

Horváth L, Kokoszka P (2012) Inference for functional data with applications. Springer, Berlin

Hsing T, Eubank R (2015) Theoretical foundations of functional data analysis, with an introduction to linear operators. Wiley, New Jersey

Jeong J, Jun M (2015) A class of Matérn-like covariance functions for smooth processes on a sphere. Spat Stat 11:1-18 
Jun M (2014) Matérn-based nonstationary cross-covariance models for global processes. J Multivar Anal 128:134-146

Leonenko NN, Taqqu MS, Terdik GH (2018) Estimation of the covariance function of Gaussian isotropic random fields on spheres, related Rosenblatt-type distributions and the cosmic variance problem. Electron J Stat 12(2):3114-3146

Marinucci D, Peccati G (2011) Random fields on the sphere: representation, limit theorems and cosmological applications. London Mathematical Society Lecture Note Series. Cambridge University Press, Cambridge

Marinucci D, Peccati G (2013) Mean-square continuity on homogeneous spaces of compact groups. Electron Commun Probab. https://doi.org/10.1214/ECP.v18-2400

Mas A (2002) Weak convergence for the covariance operators of a Hilbertian linear process. Stoch Process Appl 99(1):117-135

Michel V (2013) Lectures on constructive approximation. Springer, Berlin

Panaretos VM, Tavakoli S (2013) Cramér-Karhunen-Loève representation and harmonic principal component analysis of functional time series. Stoch Process Appl 123(7):2779-2807

Panaretos VM, Tavakoli S (2013) Fourier analysis of stationary time series in function space. Ann Stat 41(2):568-603

Porcu E, Alegría A, Furrer R (2018) Modeling temporally evolving and spatially globally dependent data. Int Stat Rev 86(2):344-377

Porcu E, Bevilacqua M, Genton M (2016) Spatio-temporal covariance and cross-covariance functions of the great circle distance on a sphere. J Am Stat Assoc 111:888-898

Porcu E, Furrer R, Nychka D (2021) 30 years of space-time covariance functions. WIREs Comput Stat 13(2):e1512

Ramsay JO, Silverman BW (2005) Functional data analysis, 2nd edn. Springer, Berlin

Schoenberg IJ (1942) Positive definite functions on spheres. Duke Math J 9:96-108

Szegö G (1975) Orthogonal polynomials. In: American mathematical society colloquium publications vol 23, 4th edn. American Mathematical Society, Providence

van Delft A, Eichler M (2020) A note on Herglotz's theorem for time series on function spaces. Stoch Process Appl 130(6):3687-3710

White P, Porcu E (2019) Towards a complete picture of stationary covariance functions on spheres cross time. Electron J Stat 13(2):2566-2594

Publisher's Note Springer Nature remains neutral with regard to jurisdictional claims in published maps and institutional affiliations. 